\title{
Electromagnetic Waves and Their Application to Charged Particle Acceleration
}

\author{
Hitendra K. Malik
}

Additional information is available at the end of the chapter

http://dx.doi.org/10.5772/52246

\section{Introduction}

A wave is a disturbance that propagates through space and time, usually with the transference of energy from one point to another without permanent displacement of particles of the medium. The particles under this situation only oscillate about their equilibrium positions. If the particles oscillate in the direction of wave propagation, then the wave is called longitudinal wave. However, if these oscillations take place in perpendicular direction with the direction of wave propagation, the wave is said to be transverse in nature. Electromagnetic (EM) waves are transverse in nature. In electromagnetic waves such as light waves, it is the changes in electric field and magnetic field that represent the wave disturbance. The propagation of the wave is described by the passage of a waveform through the medium with a certain velocity called the phase (or wave) velocity. However, the energy is transferred at the group velocity of the waves making the waveform. Electromagnetic radiation is a form of energy exhibiting wave like behavior as it travels through the space. The electromagnetic radiation is classified based on the frequency of its wave. Figure 1 shows the electromagnetic spectrum that consists of radio waves, microwaves, infrared (IR) radiation, visible light, ultraviolet (UV) radiation, $X$-rays and gamma rays. T-rays shown in the spectrum represent the terahertz $(\mathrm{THz})$ radiations. This region of frequency $\left(10^{11} \mathrm{~Hz}\right.$ to $\left.\sim 10^{13} \mathrm{~Hz}\right)$ had remained the last unexplored region between long wavelength and visible electromagnetic radiation for a long time due to the lack of efficient emitters and receptors. Interestingly this region of the $\mathrm{THz}$ rays demarcates the regions of most fascinating subjects of electronics and photonics.

\section{Propagation of electromagnetic waves}

In order to study the propagation of wave, we first let the sinusoidal variation of oscillating quantities as $\sim e^{i(\vec{k} \cdot \vec{r}-\omega t)}$ that are associated with the wave. Here $\vec{k}$ is the wave vector that 
tells about the direction of wave propagation and also gives the wavelength of oscillations as $\lambda=2 \pi / k$ and $\omega$ is the angular frequency of the oscillations that gives rise to the time period $T=2 \pi / \omega$. The relation between $\omega$ and $k$ is called the dispersion relation, based on which the wave propagation is investigated in a medium. The propagation of electromagnetic waves in any medium can be understood based on the fundamental equations of electromagnetic wave theory, i.e. the Maxwell's equations, which were established by James Clerk Maxwell in 1873 and experimentally verified by Heinrich Hertz in 1888. These Maxwell's equations are

$$
\begin{gathered}
\vec{\nabla} \cdot \vec{D}=\rho \\
\vec{\nabla} \cdot \vec{B}=0 \\
\vec{\nabla} \times \vec{E}=-\frac{\partial \vec{B}}{\partial t} \\
\vec{\nabla} \times \vec{H}=\vec{J}+\frac{\partial \vec{D}}{\partial t}
\end{gathered}
$$

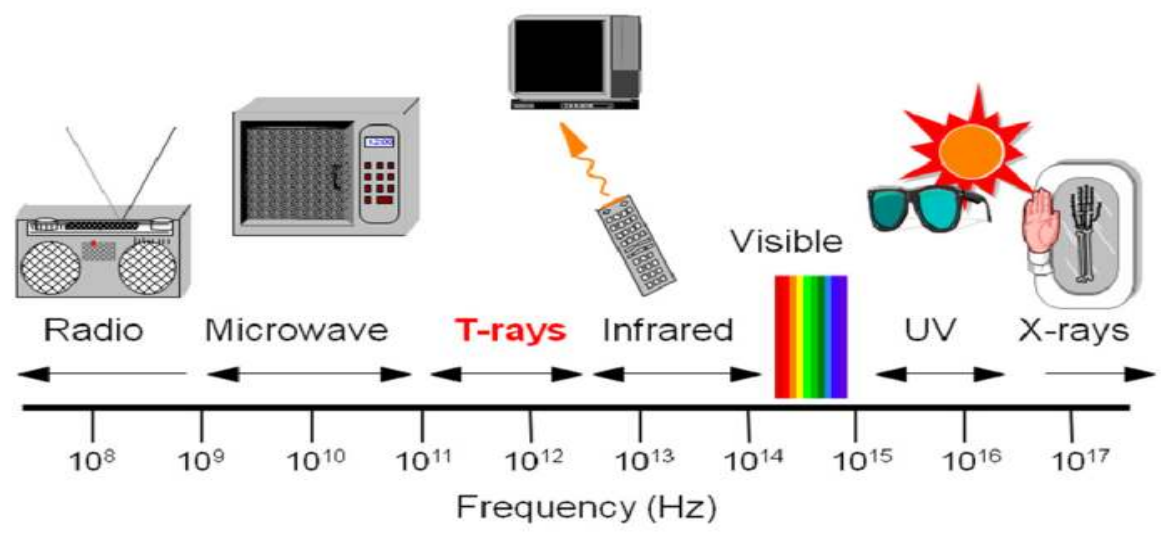

Figure 1. Electromagnetic spectrum.

Here $\vec{D}, \vec{B}, \vec{E}, \vec{H}, \vec{J}$ and $\rho$ are respectively the electric displacement $\left(\mathrm{C} / \mathrm{m}^{2}\right)$, magnetic flux density $\left(\mathrm{Wb} / \mathrm{m}^{2}\right)$, electric field strength $(\mathrm{V} / \mathrm{m})$, magnetic field strength $(\mathrm{A} / \mathrm{m})$, electric current density $\left(\mathrm{A} / \mathrm{m}^{2}\right)$ and electric charge density $\left(\mathrm{C} / \mathrm{m}^{3}\right)$, which are the real functions of position and time. For an isotropic medium $\vec{D}=\varepsilon \vec{E}$, i.e. the vector $\vec{E}$ is parallel to $\vec{D}$, and $\vec{B}=\mu \vec{H}$, i.e. the vector $\vec{H}$ is parallel to $\vec{B}$. Here $\varepsilon$ is the electric permittivity of the medium that tells about the polarization of the medium and is determined by the electrical properties of the medium. $\mu$ is the permeability of the medium that tells about the magnetization of the medium and is determined by the magnetic properties of the medium. Hence, the 
properties of the medium associated with the Maxwell's equations affect the electromagnetic wave propagation. Below we discuss the propagation of electromagnetic waves in different media.

\subsection{EM wave propagation in free space / vacuum}

For free space or vacuum $\varepsilon=\varepsilon_{0}, \mu=\mu_{0}, \vec{J}=0$ and $\rho=0$. If we put these values in Eqs.(1) (4) and take the curl of Eq.(3), we get

$$
\vec{\nabla}(\vec{\nabla} \cdot \overrightarrow{\mathrm{E}})-\nabla^{2} \overrightarrow{\mathrm{E}}=-\mu_{0} \frac{\partial}{\partial t}(\vec{\nabla} \times \overrightarrow{\mathrm{H}})
$$

The use of Eqs.(4) and (1) in Eq.(5) yields the following de-coupled equation in $\vec{E}$

$$
\nabla^{2} \overrightarrow{\mathrm{E}}-\mu_{0} \varepsilon_{0} \frac{\partial^{2} \overrightarrow{\mathrm{E}}}{\partial t^{2}}=0
$$

Similarly the wave equation for the field $\vec{H}$ is obtained as

$$
\nabla^{2} \overrightarrow{\mathrm{H}}-\mu_{0} \varepsilon_{0} \frac{\partial^{2} \overrightarrow{\mathrm{H}}}{\partial t^{2}}=0
$$

Equations (6) and (7) describe respectively the propagation of the fields $\vec{E}$ and $\vec{H}$ in free space. For the plane uniform wave, following are the solutions of these second order homogeneous differential equations

$$
\overrightarrow{\mathrm{E}}(\vec{r}, t)=\overrightarrow{\mathrm{E}}_{0} e^{i(\vec{k} \cdot \vec{r}-\omega t)} \text { and } \overrightarrow{\mathrm{H}}(\vec{r}, t)=\overrightarrow{\mathrm{H}}_{0} e^{i(\vec{k} \cdot \vec{r}-\omega t)}
$$

The above solutions should satisfy the respective wave equations. For example, when we put the solution for $\vec{E}$ in Eq.(6) and replace $\vec{\nabla}$ by $i \vec{k}$ and $\frac{\partial}{\partial t}$ by $-i \omega$, we get for harmonic wave with single frequency

$$
\left(k^{2}-\omega^{2} \mu_{0} \varepsilon_{0}\right) \overrightarrow{\mathrm{E}}=0
$$

Since $\vec{E}$ cannot be zero for the existence of wave, the wave equation will be satisfied only if

$$
k^{2}-\omega^{2} \mu_{0} \varepsilon_{0}=0
$$

This is the dispersion relation of the electromagnetic wave in free space or vacuum. The ratio of $\omega$ and $k$ gives rise to the phase velocity (say $v$ ) of the wave, i.e.

$$
v=\frac{\omega}{k}=\frac{1}{\sqrt{\mu_{0} \varepsilon_{0}}}=3 \times 10^{8} \mathrm{~m} / \mathrm{sec}=c \text {, the speed of light. }
$$


Hence, it is clear that the electromagnetic wave propagates with the speed of light in free space.

In addition, we can examine the nature of the electromagnetic wave based on the directions of the fields $\vec{E}, \vec{H}$ and the wave vector $\vec{k}$. The use of solution (8) in Eq.(3) yields

$$
\vec{k} \times \overrightarrow{\mathrm{E}}=\omega \mu_{0} \overrightarrow{\mathrm{H}}
$$

Similarly we obtain from Eq.(4)

$$
\vec{k} \times \overrightarrow{\mathrm{H}}=\omega \varepsilon_{0} \overrightarrow{\mathrm{E}}
$$

Equation (11) says that the field vector $\vec{H}$ is perpendicular to both $\vec{k}$ and $\vec{E}$ vectors. Also the vector $\vec{E}$ is perpendicular to both $\vec{k}$ and $\vec{H}$ vectors [see Eq.(12)]. When we combine both the equations (11) and (12), it is inferred that the vectors $\vec{E}, \vec{H}$ and $\vec{k}$ form a set of orthogonal vectors such that the cross product of $\vec{E}$ and $\vec{H}$ is always in the direction of $\vec{k}$. For this reason, the energy associated with the electromagnetic waves is carried in the direction of wave propagation. On the other hand, Eq.(1) reveals that $\vec{k} \cdot \vec{E}=0$ whereas Eq.(2) yields $\vec{k} \cdot \vec{H}=0$. It means the oscillations of the electric field $\vec{E}$ are perpendicular to the direction of wave propagation; the same is the case with the magnetic field. Hence, it is evident that the electromagnetic waves are transverse in nature.

\subsection{EM wave propagation in a dielectric}

In an isotropic dielectric medium, the current density $\vec{J}$ and volume charge density $\rho$ are zero. Also the vectors $\vec{D}$ and $\vec{B}$ are defined as $\vec{D}=\varepsilon_{0} \vec{E}+\vec{P} \equiv \varepsilon \vec{E}$ and $\vec{B}=\mu_{0} \vec{H}+\mu_{0} \vec{M} \equiv \mu \vec{H}$ for the isotropic linear dielectric medium, which is polarizable and magnetic. Here the vectors $\vec{P}$ and $\vec{M}$ give respectively the measure of polarization and magnetization of the medium. Nonetheless, for the dielectric medium it would be sufficient to remember that $\varepsilon_{0}$ and $\mu_{0}$ of free space have been replaced with $\varepsilon$ and $\mu$. Hence, for the dielectric medium the Maxwell's equations (1) - (4) take the form

$$
\begin{gathered}
\vec{\nabla} \cdot \overrightarrow{\mathrm{E}}=0 \\
\vec{\nabla} \cdot \overrightarrow{\mathrm{H}}=0 \\
\vec{\nabla} \times \overrightarrow{\mathrm{E}}=-\mu \frac{\partial \overrightarrow{\mathrm{H}}}{\partial t} \\
\vec{\nabla} \times \overrightarrow{\mathrm{H}}=-\varepsilon \frac{\partial \overrightarrow{\mathrm{E}}}{\partial t}
\end{gathered}
$$


Following the similar procedure as done in the case of free space, the wave equations for the fields $\vec{E}$ and $\vec{H}$ are obtained as

$$
\nabla^{2} \vec{E}=\mu \varepsilon \frac{\partial^{2} \vec{E}}{\partial t^{2}}, \nabla^{2} \vec{H}=\mu \varepsilon \frac{\partial^{2} \vec{H}}{\partial t^{2}}
$$

A comparison of these wave equations with Eqs.(6) and (7) reveals that the phase velocity $v$ of the wave in a linear dielectric medium is

$$
v=\frac{1}{\sqrt{\mu \varepsilon}}=\frac{1}{\sqrt{\mu_{0} \mu_{\mathrm{r}} \varepsilon_{0} \varepsilon_{\mathrm{r}}}}=\frac{c}{\sqrt{\mu_{\mathrm{r}} \varepsilon_{\mathrm{r}}}}
$$

From the above equation, it is clear that the propagation velocity of an electromagnetic wave in a dielectric medium is less than that in free space. Also the refractive index, say $n$, can be evaluated as $n=\frac{c}{v}=\sqrt{\mu_{r} \varepsilon_{r}}$. Since for a non-magnetic dielectric medium $\mu_{r} \approx 1$, the refractive index is simply given by square root of the relative permittivity, i.e. $n \approx \sqrt{\varepsilon_{r}}$. This is also true for most materials as for them $\mu \approx \mu_{0}$ and hence $\mu_{r} \approx 1$.

\subsection{EM wave propagation in a conductor}

We consider a conducting linear and isotropic medium whose permeability is $\mu$, permittivity is $\varepsilon$ and the conductivity is $\sigma$. In the cases of vacuum and dielectrics or insulators, the conductivity is zero and hence the current density $\vec{J}$ was neglected in the Maxwell's equations. Moreover, the free charge density $\rho$ was taken to be zero in these cases. In the case of conductors, the flow of charge however is not independently controlled and the current density in general cannot be neglected. Since any free charge supplied to a conductor gets dissipated, we can rather take $\rho=0$. This can be seen based on the continuity equation $\frac{\partial \rho}{\partial t}+\vec{\nabla} \cdot \vec{J}=0$. The use of Ohm's law $\vec{J}=\sigma \vec{E}$ and Gauss law of electricity $\vec{\nabla} \cdot \vec{D}=\rho$ in this equation leads $\frac{1}{\rho} \frac{\partial \rho}{\partial t}=-\frac{\sigma}{\varepsilon}$, the integration of which gives $\rho(t)=\rho(0) e^{-\frac{\sigma}{\varepsilon} t}$ together with $\rho(0)$ as the initial free charge density. This relation shows that if we put some free charge on a conductor, it will flow out to the edges in a characteristic time $\tau_{f} \equiv \frac{\varepsilon}{\sigma}$. For a perfect conductor this characteristic time $\tau_{f}=0$ as $\sigma=\infty$, and for a good conductor $\tau_{f}$ will be much less than the other relevant times, for example $\frac{1}{\omega}$ in an oscillatory system, i.e. $\tau_{f}<<\frac{1}{\omega}$. Under this situation, we can write the Maxwell's equations as

$$
\vec{\nabla} \cdot \overrightarrow{\mathrm{E}}=0
$$




$$
\begin{gathered}
\vec{\nabla} \cdot \overrightarrow{\mathrm{H}}=0 \\
\vec{\nabla} \times \overrightarrow{\mathrm{E}}=-\frac{\partial \overrightarrow{\mathrm{B}}}{\partial t}=-\mu \frac{\partial \overrightarrow{\mathrm{H}}}{\partial t} \\
\vec{\nabla} \times \overrightarrow{\mathrm{H}}=\sigma \overrightarrow{\mathrm{E}}+\varepsilon \frac{\partial \overrightarrow{\mathrm{E}}}{\partial t}
\end{gathered}
$$

Taking the curl of Eq.(21) and then making use of Eqs. (19) and (22), we get the following electromagnetic wave equation for the field $\vec{E}$ in a conductor

$$
\nabla^{2} \overrightarrow{\mathrm{E}}=\mu \sigma \frac{\partial \overrightarrow{\mathrm{E}}}{\partial t}+\mu \varepsilon \frac{\partial^{2} \overrightarrow{\mathrm{E}}}{\partial t^{2}}
$$

Similarly the wave equation for the field $\vec{H}$ is obtained as

$$
\nabla^{2} \overrightarrow{\mathrm{H}}=\mu \sigma \frac{\partial \overrightarrow{\mathrm{H}}}{\partial t}+\mu \varepsilon \frac{\partial^{2} \overrightarrow{\mathrm{H}}}{\partial t^{2}}
$$

In one-dimension (along $\mathrm{z}$-axis) the wave equations are written as

$$
\begin{aligned}
& \frac{\partial^{2} \vec{E}}{\partial z^{2}}=\mu \sigma \frac{\partial \vec{E}}{\partial t}+\mu \varepsilon \frac{\partial^{2} \vec{E}}{\partial t^{2}} \\
& \frac{\partial^{2} \vec{H}}{\partial z^{2}}=\mu \sigma \frac{\partial \vec{H}}{\partial t}+\mu \varepsilon \frac{\partial^{2} \vec{H}}{\partial t^{2}}
\end{aligned}
$$

If we compare Eq.(23) with Eq.(6), we notice that an additional term $\mu \sigma \frac{\partial \vec{E}}{\partial t}$ appears in the wave equation for the $\vec{E}$ field; the same is the case with Eq.(24) and an additional term $\mu \sigma \frac{\partial \vec{H}}{\partial t}$ appears. Hence, these wave equations are called modified wave equations for the electromagnetic field in a conductor. Owing to the inclusion of conductivity $\sigma$, both the additional terms are called the dissipative terms as these allow the current to flow through the medium.

We can assume the following plane wave solution (in one-dimension) to the wave equations (25) and (26)

$$
\vec{E}(z, t)=\vec{E}_{0} e^{i(k z-\omega t)} \text { and } \vec{H}(z, t)=\vec{H}_{0} e^{i(k z-\omega t)}
$$

Putting the above solution of $\vec{E}$ in Eq.(25) or of $\vec{H}$ in Eq.(26) we get

$$
k^{2}=\mu \varepsilon \omega^{2}+i \mu \sigma \omega
$$


This relation shows that the wave vector is a complex quantity, say $k \equiv k_{r}+i k_{i}$. With this the fields $\vec{E}$ and $\vec{H}$ become

$$
\vec{E}(z, t)=\vec{E}_{0} e^{-k_{i} z} e^{i\left(k_{r} z-\omega t\right)} \text { and } \vec{H}(z, t)=\vec{H}_{0} e^{-k_{i} z} e^{i\left(k_{r} z-\omega t\right)}
$$

It is evident from the above expressions that when the electromagnetic wave propagates through a conductor, its amplitude decreases and hence the attenuation of the wave takes place. The distance through which the amplitude is reduced by a factor of $1 / e$ is called skin depth (say $\delta$ ). The skind depth is decided by the imaginary part of the wave vector, i.e. $k_{i}$, as it can be seen that

$$
\delta \equiv \frac{1}{\left|k_{i}\right|}
$$

The real part of the wave vector determines the wavelength, phase velocity, and the refractive index in the usual manner, i.e. $\lambda=\frac{2 \pi}{k_{r}}, v=\frac{\omega}{k_{r}}$ and $n=\frac{c}{v}=\frac{c k_{r}}{\omega}$. Putting $k=k_{r}+i k_{i}$ in Eq.(28) we obtain

$$
k_{r}=\omega \sqrt{\frac{\mu \varepsilon}{2}}\left\{\sqrt{1+\left(\frac{\sigma}{\omega \varepsilon}\right)^{2}}+1\right\} \text { and } k_{i}=\omega \sqrt{\frac{\mu \varepsilon}{2}}\left\{\sqrt{1+\left(\frac{\sigma}{\omega \varepsilon}\right)^{2}}-1\right\}
$$

It is evident that the propagation of the wave and the skin depth depend on the properties of the conductor and the frequency of the wave. Based on the expression of $k_{i}$, this can be seen that the skin depth for the electromagnetic waves having high frequencies is smaller. Since the skin depth is a measure of how far the wave penetrates into the conductor, the high frequency waves are found to penetrate less into the conductor. For example, in the case of copper, the skin depth of approximately $6 \mathrm{~cm}$ is obtained at the frequency of $1 \mathrm{~Hz}$ and it decreases to about $2 \mathrm{~mm}$ if the frequency is increased to $1 \mathrm{KHz}$. The skin depth causes the effective resistance of the conductor to increase at higher frequencies where the skin depth is smaller, thus reducing the effective cross-section of the conductor. If we talk in general about the skin depth, it is the tendency of an alternating electric current to distribute itself within a conductor with the largest current density near the surface of the conductor and decreased density at greater depths. Under this situation, the electric current flows mainly at the skin of the conductor. Hence, the word skin comes into picture.

During the wave propagation in conductors, unlike the cases of vacuum and dielectrics, the electric field and magnetic field vectors do not remain in phase. This can be seen as follows. Taking the direction of electric field $\vec{E}$ along the $x$-axis, we write it as $\vec{E}(z, t)=\hat{x} E_{0} e^{-k_{i} z} e^{i\left(k_{r} z-\omega t\right)}$. Using this in Eq.(21), we get $\vec{H}(z, t)=\hat{y} \frac{k E_{0}}{\omega \mu} e^{-k_{i} z} e^{i\left(k_{r} z-\omega t\right)}$. Clearly the amplitude of field $\vec{H}$ contains $k$, which is a complex quantity and can be expressed in 
terms of its magnitude (say $k^{\prime}$ ) and phase (say $\theta_{k}$ ) as $k=k^{\prime} e^{i \theta_{k}}$. Here $k^{\prime}=\sqrt{k_{r}^{2}+k_{i}^{2}}=\omega \sqrt{\mu \varepsilon \sqrt{\left\{1+\left(\frac{\sigma}{\varepsilon \omega}\right)^{2}\right\}}}$ and $\theta_{k}=\tan ^{-1}\left(\frac{k_{i}}{k_{r}}\right)$. With this the field can be written as $\vec{H}(z, t)=\hat{y} \frac{k^{\prime} E_{0}}{\omega \mu} e^{-k_{i} z} e^{i\left(k_{r} z-\omega t+\theta_{k}\right)}$. A comparison of this expression with $\vec{E}(z, t)=\hat{x} E_{0} e^{-k_{i} z} e^{i\left(k_{r} z-\omega t\right)}$ clearly infers that $\theta_{H}-\theta_{E}=\theta_{k}$, where $\theta_{H}$ is the phase of the magnetic field and $\theta_{E}$ is the phase of the electric field. Hence, the magnetic field lags behind the electric field during the electromagnetic wave propagation in a conductor.

\section{EM waves and plasma interaction}

Our aim is to disucss the electromagnetic waves and plasma interaction in view of the particle acceleration. Hence, now we introduce the plasma as a new medium, which is sometimes referred to as the fourth state of the matter.

\subsection{Plasma: Fourth state of matter}

Everybody is well aware of three states of the matter, i.e. the solid, liquid and gas. In solids, the atoms are packed very close to each other and are fixed at definite positions. These are connected with each other by the interatomic forces. The atoms of solids start oscillating about their equilibrium positions when we supply energy to them, and as a result the interatomic forces become weaker and the atoms are separated significantly. This way the solid takes the form of liquid, where the atoms or molecules override. The liquid has a specific volume but does not have precise shape. So it changes shape according to the shape of the container in which it is kept. If we further supply energy to the atoms, the interatomic forces become insignificant, the atoms get separated and start moving freely. Under this situation, the liquid takes the form of gas. In gas, the atoms are not connected with each other and hence can move in any direction. The gas neither has precise shape nor the fixed volume. It takes the shape and volume of the container in which it is kept. If more energy is supplied to the atoms (or molecules) of a gas, the electrons from the outermost level of the atoms get easily detached and hence the atoms become ionized. As a result, we are left with the collection of ions, electrons and some neutrals (unionized atoms). This collection of charged and neutral particles is referred to as plasma. This is sometimes called the fourth state of matter, as it is found in natural conditions. For example, the gases near the sun are always in ionized state that qualifies for plasma. The species of the plasma being charged are connected with each other by the electromagnetic forces. This can be understood as follows. Since the charges separated with each other set up the electric field, the plasma species produce the electric field. However, the separation of charges of plasma is not fixed (as the species do not remain stationary). So this electric field is time varying field, which will generate magnetic field according to the Maxwell's fourth equation. On the other hand, the motion of charges generates current and 
hence the magnetic field. In view of this, the plasma species produce time varying magnetic field that will induce electric field according to the Maxwell's third equation. Thus, it can be said that the plasma species are connected with each other by the electromagnetic fields. In view of almost equal number of ions and electrons in the plasma, the plasma as a whole is neutral. However, the plasma is quasineutral, as we cannot neglect the internal forces at the same time. Moreover, if we attempt to disturb a part of the plasma, the whole body of the plasma gets perturbed due to the connection of all the species with each other. This property is known as collective behaviour of the plasma. Therefore, an ionized gas can qualify for plasma state, if it is quasineutral and it shows collective behaviour.

Another interesting property of the plasma is its ability to shield out the field that is applied on it. This can be better understood, for example, when we insert the electrodes of a battery into the plasma. Then the positive (negative) electrode attracts the electrons (ions) whose number is decided by the charge carried by the electrode. So an electron cloud is developed around the electrode that shields / cancels the external field. The thickness of this electron cloud is known as Debye length (say, $\lambda$ De). Since the electrons are light species compared with ions, the shielding is generally accomplished by the electrons only. It is clear that the field exists within the cloud or the Debye sphere (sphere with the radius $\lambda \mathrm{De}$ ). Now imagine if the Debye length is much less than the dimension $(\mathrm{L})$ of the plasma. Then the bulk of the plasma will remain neutral. Therefore, $\lambda \mathrm{De} \ll \mathrm{L}$ is the required condition for the quasineutrality. In aadtion, if the number of electrons in the Debye sphere (say, NDe) is much larger than unity, i.e. $\mathrm{NDe}_{\mathrm{D}} \gg$, then the condition of collective behaviour will be fulfilled. Any distance in the plasma system is measured in terms of Debye length $\lambda$ De and the time is measured in terms of reciprocal of plasma frequency (say $f_{p e}$ ). The plasma frequency is nothing but the natural frequency of the plasma, the same as all the materials have their natural frequencies. Actually this is the frequency of oscillations made by the electrons about their equilibrium positions. The plasma frequency $f_{p e}$ and the Debye length $\lambda$ De in SI system of units are given by

$$
f_{p e}=\frac{1}{2 \pi}\left(\frac{n_{0} e^{2}}{\varepsilon_{0} m_{e}}\right)^{1 / 2} \text { and } \lambda_{D e}=\left(\frac{\varepsilon_{0} k T_{e}}{n_{0} e^{2}}\right)^{1 / 2}
$$

In these expressions, $n_{0}$ is the plasma density, which is the common density of ions $\left(n_{\mathrm{i}}\right)$ and electrons $\left(n_{\mathrm{e}}\right)$, i.e. $n_{0}=n_{\mathrm{i}}=n_{\mathrm{e}}, T_{e}$ is the electron temperature, $k$ is the Boltzmann constant $\left(k=1.38 \times 10^{-23} \mathrm{~J} / \mathrm{K}\right), e$ is the electronic charge and $m_{\mathrm{e}}$ is the electron mass. In plasmas, generally we do not talk about the temperature of the ions and electrons, but we specifically focus on their energies. It means the temperature is written in terms of the energy. For example, $1 \mathrm{eV}$ energy of the electron would be equal to its thermal energy $k T_{e}$ (for twodimensional system, and in general in plasma physics). So

$$
\begin{array}{ll} 
& 1 \mathrm{eV}=\mathrm{kT}_{\mathrm{e}} \\
\text { or } & 1.6 \times 10^{-19}(J)=1.38 \times 10^{-23} \mathrm{~T}_{\mathrm{e}}(\mathrm{J} / \mathrm{K}) \\
\text { or } & \mathrm{T}_{\mathrm{e}}=11,600 \mathrm{~K} .
\end{array}
$$


It means $1 \mathrm{eV}$ energy is equivalent to $11,600 \mathrm{~K}$ temperature. The electron temperature in laboratory plasmas generally varies from $1 \mathrm{eV}$ to $5 \mathrm{eV}$. For a plasma with number density of $10^{18} / \mathrm{m}^{3}$ and temperature $2 \mathrm{eV}$, the Debye length comes out to be of the order of $\mu \mathrm{m}$ and the plasma frequency of the order of $\mathrm{GHz}\left(10^{9} \mathrm{~Hz}\right)$.

Hence, it is clear that only the electrons would be able to respond to the high frequency field of the electromagnetic waves, for example, microwaves or lasers. As mentioned, our aim is to develop an understanding for the electromagnetic waves and plasma interaction for their possible applications to the particle acceleration. Below we discuss about this topic in greater detail and summarize the research conducted in this direction. At first we talk about the phenomena that may be realized during the interaction of electromagnetic waves and plasmas.

\subsection{Some basic phenomena}

According to linear theory, only the electromagnetic wave of frequency $\omega$ higher than plasma frequency $\omega_{p e}$ can propagate through the plasma. The wave whose frequency $\omega$ is below $\omega_{p e}$ gets reflected and the one with $\omega=\omega_{p e}$ gets absorbed resonantly in collisionless plasma. The plasma itself can support several types of electrostatic and electromagnetic waves such as electron plasma wave, ion acoustic wave, electron electromagnetic waves, etc. The interaction of electromagnetic wave with plasma can take place through the exciation of such waves and in this process the exchange of energy can be possible between the electromagnetic wave and plasma species. If the amplitude of wave is much higher than its nonlinear interaction with other collective modes in plasma, plasma instabilities are dominant. On the other hand, the wave can also decay by Landau damping and if plasma is underdense $\left(\omega>\omega_{p e}\right)$ then the wave can decay in electrostatic wave and some other electromagnetic waves, resulting in parametric instabilities including Raman scattering, Brillouin scattering, etc. In case of large amplitude wave, the effect of ponderomotive force also comes into picture. This is very important phenomenon in view of harmonic generation, beat wave excitation, wakefield excitation for particle acceleration, self-focusing of laser beam, filamentation of laser beam, etc.

In the theory for resonance absorption, wave propagation in the resonance layer is described either by electron-ion collisions and thermal dispersion or by nonlinear effects like wave breaking, etc. [1 - 3]. The anomalous absorption of electromagnetic waves on a surface of an inhomogeneous unmagnetized plasma was theoretically predicted by Gildenburg [4]. Later this phenomenon was confirmed experimentally, out of which some experiments have shown that large amount of power can be absorbed by magnetized plasma at the electron and ion cyclotron frequencies. A usual way of coupling transverse waves into a plasma for the purpose of such resonant absorption has been to use a magnetic beach as suggested by Stix [5]. Breizman et al. [6] have presented a selfconsistent theory of the rf-wave propagation and ion motion through the resonance. An important ingredient of the problem is the ion flow along the magnetic field. The flow 
velocity limits the time the ions spend at the resonance, which in turn limits the ion energy gain. A feature that makes the problem nonlinear is that the flow accelerates under the effect of $\vec{\nabla} B$ force and rf-pressure. This acceleration can produce a steep reduction in the plasma density at the resonance, resulting in partial reflection of the incident wave. The propagation and collisionless absorption of electromagnetic waves propagating in nonuniformly magnetized plasmas with regions of cyclotron resonance were computed by Kuckes [7]. He considered the particle dynamics associated with motion in a nonuniform magnetic field near cyclotron resonance explicitly and predicted the complete wave absorption above a critical plasma density.

The nonlinear behaviour of the large-amplitude plasma wave and the effect of an inhomogeneous plasma on its growth and saturation in a collisionless plasma due to the beating of two laser beams with frequencies much above the plasma frequency $\omega_{p e}$ has been considered taking into account the modulation of the Lorentz force by the large-amplitude plasma wave as well as the temporal variation of its phase [8]. In this case, a novel parametric instability as a result of the modulation of the Lorentz force by the largeamplitude plasma wave is found when the beat frequency is twice the plasma frequency. The high phase velocity electron plasma wave excited by collinear optical mixing has been detected directly [9], where the frequency, wave number, spatial extent, saturation time, and peak amplitude were all measured experimentally and found to be in reasonable agreement with the theoretical expectations. The resonant excitation of an electron plasma wave and its effects on the density profile steepening have been theoretically studied by using a modified, warm-capacitor model [10], where the scaling laws characterizing the process were established and the wave structure and density profile were self-consistently determined.

Chang et al. [11] have observed experimentally the parametric excitation of ion acoustic waves and cyclotron harmonic waves by a high frequency electric field with frequencies near the harmonics of the cyclotron frequency. They have verified both the wave vector and the frequency selection rules. Parametric excitation of longitudinal oscillations of plasma was studied by Kitsenko et al. [12] in a weak alternating electric field with frequency $\omega_{0}$ close to that of electron-ion hydrodynamic longitudinal oscillations of cold plasma, $\omega_{1}=\omega_{L H} \sqrt{\left[1+\left(m_{i} / m_{e}\right) \cos ^{2} \theta\right]}$, where $\omega_{L H}$ is the lower hybrid resonance frequency. In their study, the angle $\theta$ between the direction of propagation of the oscillations and the magnetic field was close to $\pi / 2$ and it was shown that oscillations can be excited in the plasma with frequencies much less than $\omega_{0}$, if the drift velocity of the particles in the steady external magnetic field and the alternating electric field is less than the thermal velocity of the ions.

Optical investigations have been reported of the interaction of $0.3 \mathrm{TW}, 250 \mathrm{fs}$ Ti: sapphire laser pulses with underdense plasmas created from high density gas jet targets [13]. Time resolved shadowgraphy using a $2 \omega$ probe pulse, images of the transmitted radiation and images of $1 \omega$ and $2 \omega$ side radiations were presented for various gases. Their experimental 
results and analysis based on a simple numerical Gaussian beam model showed that ionization-induced refraction dominates the interaction process for all gases except hydrogen. The numerical modeling has also shown that for a given laser power there exists only a narrow density range in which self-focusing can be expected to occur. On the other hand, it has been observed that the nonlinear frequency shift of a strong electromagnetic wave in a plasma due to weak relativistic effects and the $\vec{v} \times \vec{B}$ force can cause modulation and self-focusing instabilities [14]. Kaw et al. [15] have shown that an electromagnetic wave interacting with a plasma is subject to instabilities that leads to light filamentation. Numerical studies of beam filamentation in laser produced plasma have been presented by Nickolas et al. [16] based on a parabolic wave equation, known as the Schroedinger equation, coupled with thermal transport equations for both the ions and electrons in twodimensions. Also the results of a numerical code have been described which models the relativistic self-focusing of high intensity laser beams in plasmas by the nonlinear relativistic dependence of the optical constants on laser intensity [17]. Here rapid relativistic selffocussing down to a beam diameter of one micron in a distance of the order of the original beam diameter was observed. They also observed the production of $\mathrm{GeV}$ ions moving against the laser light.

\subsection{Particle acceleration}

Particle accelerators are among the largest machines built by humans. In the conventional linear accelerators (LINACs), the acceleration gradients are however limited to some tens of $\mathrm{MeV} / \mathrm{m}$. Since the energy gain of particles is the product of such acceleration gradient and the acceleration distance, we need to extend only the acceleration distances in order to reach high energies. That's why these tools for high energy physics are becoming larger and larger, and increasingly more expensive. For the first time, it was realized by Tajima and Dawson [18] that a laser beam propagating in a plasma can excite electron plasma wave, which being longitudinal can be used to accelerate electrons. To understand the underline principle for plasma based acceleration, consider the limits of conventional particle accelerators based on rf-waves propagating in corrugated metallic cavities. They are limited first by the availability of high peak power drivers and ultimately by electrical breakdown of the metal structure. These factors correspond to linear accelerating gradient of $20-100 \mathrm{MeV} / \mathrm{m}$. Plasmas though are not limited by breakdown as they are already ionized and indeed can support electric fields of the order $10-100 \mathrm{GeV} / \mathrm{m}$. Consequently, with regard to the energy gain of particles in accelerators, a plasma accelerator can cut down significantly the acceleration distance to boost particles from rest to several $\mathrm{MeV}$ over a short distance (less than the millimeter range) and still provide high quality electron beam. Thus, plasma based particle accelerators opened a new and exciting field of extreme gradient (beyond $1 \mathrm{TV} / \mathrm{m}$ ). There has been a tremendous progress in recent years, due to the advances in technology, particularly by the development of compact terawatt laser systems based on the technique known as chirped-pulse amplification (CPA). 


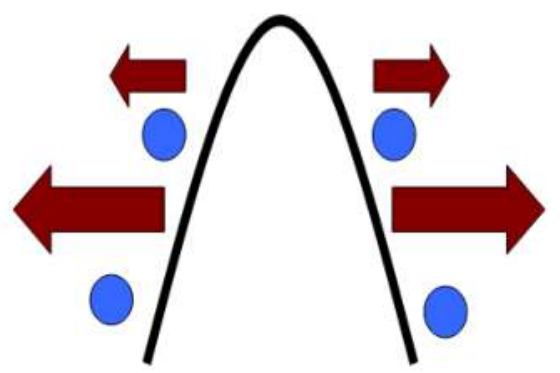

Figure 2. Laser intensity profile: Ponderomotive force.

\subsubsection{Excitation of Langmuir waves: Wakefield generation}

Although electric fields of the order of $1 \mathrm{TeV} / \mathrm{m}$ are readily achievable these days at the focus of a laser beam, these fields in vacuum cannot be used directly for the purpose of particle acceleration. This is because they are transverse and oscillatory in nature. However, if laser light can be used to excite Langmuir waves in plasma, these waves being longitudinal can be used to accelerate charged particles.

The motion of the electron in the presence of electric field is governed by the Lorentz force. In the case of high, nonuniform electromagnetic (or purely electric) field, the expression for Lorentz force has a second order term, which is proportional to the laser intensity gradient. This second order force term is known as the ponderomotive force, given by

$$
\vec{F}_{p m}=\left\{e^{2}\left(1+\alpha^{2}\right) / 4 m_{e} \omega^{2}\right\} \vec{\nabla} E^{2}
$$

Here $\omega$ is the frequency of laser having the electric field $E$ and $\alpha$ is the ellipticity of the laser light, which is equal to zero for the linearly polarized light and is unity for the circularly polarized light. The above expression is for ponderomotive force on a single electron. However, for the plasma the ponderomotive force on the electrons is defined for unit volume as per the following relation

$$
\vec{F}_{p m}=-\left(\omega_{p e} / \omega\right)^{2}\left\{\left(1+\alpha^{2}\right) / 2 c\right\} \vec{\nabla} I
$$

Thus, any spatial variation of the laser intensity $I$ will act to push the electrons / ions from the region of higher intensity to the region of lower intensity through the ponderomotive force (Fig.2). This displacement of electrons creates large amplitude plasma wave, which is called the wake. The field corresponding to this wake, i.e. the wakefield, can reach up to 100 $\mathrm{GeV} / \mathrm{m}$ provided there is a resonance between the plasma frequency ope and the ponderomotive force. The concept of wakefield acceleration can be understood based on the following example. When a speed-boat travels in water, it produces two types of waves viz. bow waves and wakefield waves. The bow waves are conical waves having tip at the front end of the boat. These are produced because the velocity of the boat exceeds that of the water waves. The wakefield waves are waves set up at the back (or wake) of the boat, which 
travels with the velocity equal to the velocity of the boat. According to the principle of the Landau damping, a floating ball dropped in the wakefield wave of the boat will get accelerated to the velocity of the boat if its initial velocity is slightly less than that of the boat. This is exactly the principle of wakefield acceleration.

Below we discuss a few methods that are used to excite plasma wave and hence the wakefield.

\subsubsection{Laser beat wave accelerator (LBWA)}

In the LBWA method, the plasma wave is excited by beating two optical waves of slightly different frequencies. Two laser waves of frequencies $\omega_{1}$ and $\omega_{2}$, having polarization in the same direction, traveling in preformed plasma of uniform density $n_{0}$ (corresponding plasma frequency $\omega_{p e}$ ) will beat at a frequency $\Delta \omega=\omega_{1}-\omega_{2}$. If this frequency difference is exactly equal to the plasma frequency (i.e. $\Delta \omega=\omega_{p e}$ ), then strong Langmuir wave will be excited in the plasma by the longitudinal ponderomotive force of the beat wave. Since the beat wave moves with the laser pulse, the plasma wave will also move with a phase velocity equal to the group velocity (near light velocity) of the laser pulses. Then a properly placed bunch of electrons with a velocity slightly lesser than the laser group velocity will get accelerated by wave-to-particle energy transfer. However, in this process there is a problem of detuning of resonance condition, which is attributed to the modified plasma frequency $\left(\omega_{p e} \propto \sqrt{1 / m_{e}}\right)$ due to the change in electron mass because of their reltivistic speeds in very large amplitude of the wakefield.

\subsubsection{Laser wakefield accelerator (LWFA)}

In beat wave acceleration scheme, it is necessary to have plasma of uniform density along with strict requirement on plasma density to exactly match with the beat wave frequency and clamping of field due to relativistic effects. Hence, laser wakefield acceleration shceme was proposed in which all the above problems are absent. For LWFA, one uses a short pulse of very high intensity. When such a high intensity laser pulse is incident on a gas, it ionizes the gas. The laser light propagates in this plasma with a velocity equal to the group velocity $\left(v_{g}\right)$ in plasma, which is nearly equal to the velocity of the light. The short laser pulse duration $\tau$ has a strong intensity variation in time and correspondingly in space. This leads to a strong longitudinal pondermotive force. The wavelength of this pondermotive force, and that of the density perturbation caused by it, is of the order of $2 c \tau$. If this is made equal to the plasma wavelength (defined as $\lambda_{p}=2 \pi c / \omega_{p e}$ ), then high amplitude wakefields are produced due to resonance (Fig.3). Similar to the case of the boat, the laser wakefield moves with the pulse at a velocity equal to the group velocity of the laser pulse. Under this situation, a correctly injected bunch of electrons can be accelerated by the longitudinal field of the plasma waves (Fig.3), where an electron bunch is injected in the plasma wave midway between every two alternate plasma wave peaks. If the plasma wave itself moves with a phase velocity $v_{p}$ and the electron beam moves with a velocity $v_{b}$, then the beam will be 
forced by the plasma wave to travel with a velocity equal to that of the plasma wave. This is because, if $v_{b}<v_{p}$, the electron bunch at point $C$ (Fig.4) will start trailing from the midpoint and will experience a positive force due to electron bunch at point $\mathrm{A}$. This will accelerate it in the $+\mathrm{z}$ direction till it attains a velocity equal to $v_{p}$. If $v_{b}>v_{p}$, then it will start drifting towards point $B$ and the bunch at $B$ will repel it backward till it slows down to a velocity equal to $v_{p}$. If the electron beam has a velocity much different from that of the plasma wave, it will cross the repulsive barriers at point A or B and its velocity will keep oscillating about its mean velocity. In other words, such a beam of electrons will not have a net exchange of energy with the plasma wave. Hence, a beam of electrons traveling with a velocity slightly less than that of the plasma wave will get accelerated. Moreover, if the phase velocity of the plasma wave is relativistic, then the slight gain in velocity corresponds to a large gain in the energy.

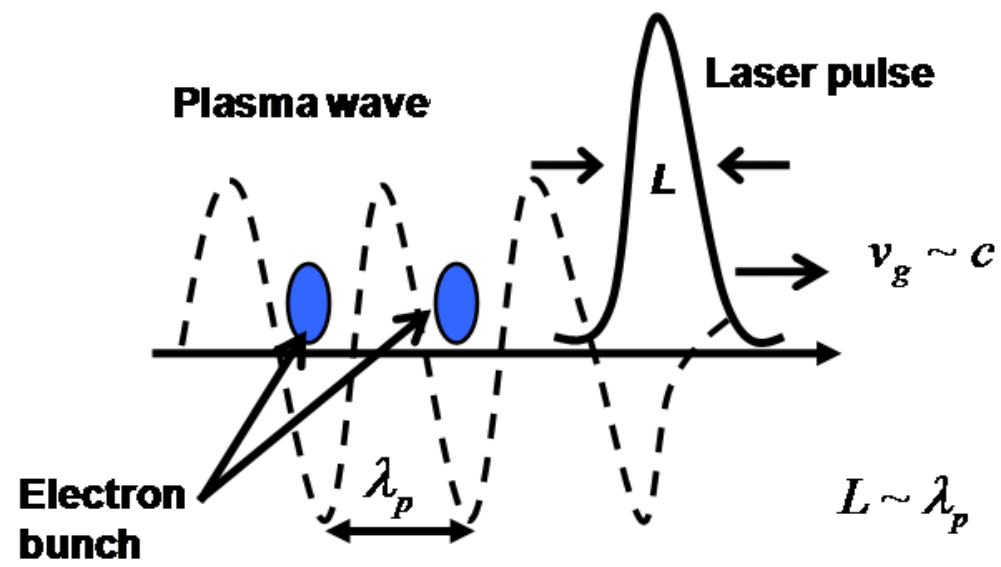

Figure 3. Schematic of LWFA.

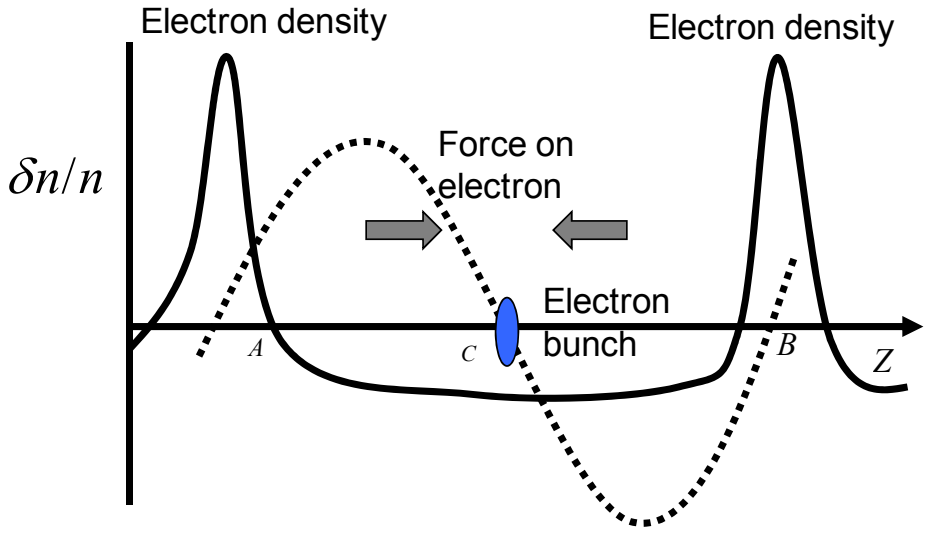

Figure 4. Force on an electron bunch trapped in an electron plasma wave. 


\subsubsection{Self modulated LWFA (SM-LWFA)}

In this scheme, the electron plasma wave is excited resonantly by the modulation of the laser pulse envelope. This occurs for a laser pulse having length $(L)$ few times longer than the plasma wavelength $\left(\lambda_{p}\right)$ and pulse power larger than the power (critical power $P_{c}$ ) required to self-focus the laser beam. Owing to the finite pulse shape, a small plasma wave is excited non-resonantly, which results in growth of forward Raman scattering (FRS) instability. The FRS wave and the laser wave beat at the plasma frequency, which gives rise to an enhancement of the electron plasma wave. Thus, there exists an oscillating density perturbation within the pulse envelope. The laser pulse therefore sees a refractive index that is alternately peaked and dented at interval of $\lambda_{p} / 2$. As the phase velocity of the laser wave depends on the density, the modulation in density gives rise to redistribution of the photon flux within the laser pulse, which leads to modulations in the envelope with a period of $\lambda_{p}$. This modulation gives rise to strong ponderomotive force with wavelength exactly equal to the plasma wavelength (as in LWFA). This strongly enhances the plasma wave amplitude. This effect grows in time, thereby transforming the initial laser pulse envelope into a train of shorter laser pulses with width of $\lambda_{p}$ or duration proportional to $1 / \omega_{p e}$. Since $\lambda_{p} \sim \sqrt{\frac{1}{n_{0}}}$ and $P_{c} \sim \frac{1}{n_{0}}$, the conditions $L>\lambda_{p}$ and $P>P_{c}$ for fixed laser parameters can usually be satisfied by operating at sufficiently high plasma density. Figure 5 shows the self-modulated scheme of laser wakefield acceleration.

The advantages of the self-modulated LWFA over the standard LWFA are the simplicity and enhanced acceleration. Simplicity is that a preformed density channel and pulse tailoring are not required for the matching condition of $L \sim \lambda_{p}$. Enhanced acceleration is achieved for the following reasons. First, the SM-LWFA operates at a higher density, which leads to a larger wakefield $\left(E \sim \sqrt{n_{0}}\right)$. Second, the wakefield is resonantly excited by a series of pulses as opposed to a single pulse in the standard LWFA, relativistic optical guiding allows the modulated pulse structure to propagate for several Rayleigh lengths. This extends the acceleration distance and hence the large energy gain is achieved in this scheme.
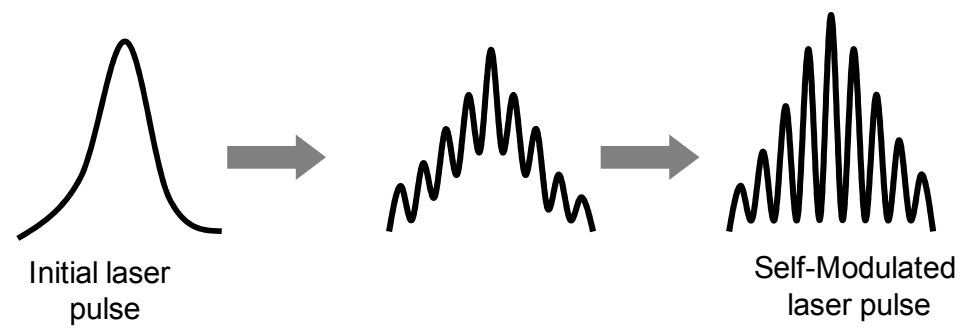

Figure 5. The self-modulated laser wakefield acceleration scheme. 
So far we have seen that when a short laser pulse propagates through underdense plasma, a large amplitude plasma wave is excited in the wake of the laser pulse by the ponderomotive force associated with the temporal profile of the pulse. For tightly focused pulses ( $k_{p} \times w_{0} \leq 1$, where $k_{p}$ and $w_{0}$ are the plasma wave vector and the beam size at the waist, respectively), both longitudinal and radial components of the ponderomotive force generate a density perturbation, whereas in loosely focusing geometry $\left(k_{p} \times w_{0}>>1\right)$, only a longitudinal electron plasma wave is generated. The amplitude of the wave is maximum when $\omega_{p e} \times \tau \sim 1$, where $\tau$ is the pulse duration and $\omega_{p e}$ is the plasma frequency.

\subsubsection{Plasma wakefield accelerator (PWFA)}

In a plasma wakefield accelerator (PWFA), the electron plasma wave is driven by one or more electron beams. Effectively the wakefield can be excited by a relativistic electron beam. This can be achieved if the electron beam terminates in a time shorter than the plasma period $1 / \omega_{p e}$. In such a scheme, the ratio of energy gain to the drive beam energy (called transformation ratio) is limited to $\leq 2$ for a symmetric driving beam in the linear regime. However, it can be increased by using an asymmetric drive beam.

\subsubsection{Studies on particle acceleration}

The researchers all over the world have made various attempts to accelerate the charged particles using wakefield and other mechanisms. Below we summarize the work done using lasers, microwaves and electron bunches.

\subsubsection{Acceleration by wakefield}

The investigations on the excitation of wakefield began with the pioneering work of Chen et al. [19], and the first experimental evidence was reported by Rosenzweig and coworkers [20, 21] followed by Nakajima et al. [22]. The wakefield generation has been widely studied experimentally, analytically and using simulations [23 - 29]. Nishida et al. [30] have successfully excited wakefield in the ion wave regime with long pulse duration by employing a variety of driving bunch shapes. Later, Aossey et al. [31] observed such type of wakefield in three-component plasma also. On the other hand, efforts have been made related to wakefield excitation by relativistic electron bunch [29], [32], and coupling of longitudinal and transverse motion of accelerated electrons in laser wakefield [25]. Lotov [24] has analytically studied the laser wakefield acceleration in narrow plasma filled channels. Analytical investigations on wakefield acceleration using a dielectric lined waveguide structure showed the acceleration gradient for electrons or positrons in the range of $50-100 \mathrm{MV} / \mathrm{m}$ for a few $\mathrm{nC}$ driving bunches [33]. In another wakefield accelerator, a peak acceleration gradient of $155 \mathrm{MeV} / \mathrm{m}$ was predicted for a $2 \mathrm{nC}$ rectangular drive bunch [34]. Jing et al. [35] have found transverse wakefield of about $0.13 \mathrm{MeV} / \mathrm{mnC}(0.2 \mathrm{MeV} / \mathrm{mnC})$ due to X-dipole modes (Y-dipole modes) in an $\mathrm{X}$-band structure generated by an electron bunch in dipole-mode wakefield in a waveguide accelerating structure. Short microwave pulses have also been used in some experiments to excite a nonlinear large amplitude ion 
wave at resonance absorption region [36]. This has also been suggested that the wakefield of an ultra short laser pulse can be amplified by a second laser pulse copropagating behind with duration of a few plasma wavelengths or longer [37]. Malik [38] has analytically investigated the wakefield in waveguide generated by the different types of microwave pulses with moderate intensities.

For the purpose of efficient acceleration, it is necessary to excite the wakefield of a large amplitude along with its speed nearly equal to the speed of light. The wakefield is reported to be enhanced by the nonlinearities in response of plasma to ponderomotive force of a long smooth laser pulse of relativistic intensity whose pulse length is much larger than the half of the plasma wavelength [39]. The amplitude of the laser wakefield has also been found to increase by the ionization processes of the gases at comparatively higher laser peak intensities [40]. A capillary tube can be used as a waveguide in order to enhance the interaction length [41]. Tapered plasma channels have been proposed for the enhancement of interaction length to achieve greater acceleration [42]. However, in such interactions, when the plasma wave acquires sufficiently large amplitude it becomes susceptible to instability, which is also an important issue in nonlinear plasma physics [43 - 53] in addition to other types of waves, structures and instabilities [54 - 63] including the laser produced plasmas [64] that may support different types of growing waves under the effect of high magnetic field [65].

\subsubsection{Acceleration using lasers}

McKinstrie and Startsev [66] have proposed that a laser field can accelerate the preaccelerated electron significantly. However, they neglected the effect of longitudinal field of the laser pulse. On the basis of 3-D particle-in-cell simulations for the ion acceleration from a foil irradiated by a laser pulse, Pukhov [67] has shown that at the front side the laser ponderomotive force pushes electrons inward and creates the electric field by charge separation, which drags the ions. Yu et al. [68] considered the electron acceleration from the interaction of an intense short pulse laser with low density plasma and the optimum condition for the acceleration in the wake was obtained. They showed that the electron acceleration within the pulse dominates as the pulse becomes sufficiently short. By using 2D particle-in-cell simulation, Suk [69] has studied the electron acceleration based on selftrapping by plasma wake. Sentoku et al. [70] examined experimentally the interaction of short laser pulse with dense plasma target for the proton acceleration and found that the peak proton energy increases in inverse proportion to the target thickness. Singh and Tripathi [71] have studied the laser induced electron acceleration in a tapered magnetic wiggler where the IFEL resonance condition was maintained for longer duration. With regard to the importance of polarization effects, Kado et al. [72] have observed strongly collimated proton beam from Tantalum targets when irradiated with circularly polarized laser pulses. With the help of radially polarized ultra relativistic laser pulses, Karmakar and Pukhov [73] have shown that collimated attosecond GeV electron bunches can be produced by ionization of high- $Z$ material. They also compared the results with the case of Gaussian laser pulses and found that the radially polarized laser pulses are superior both in the maximum energy gain and in the quality of the produced electron beams. $\mathrm{Xu}$ et al. [74] 
made a comparison between circularly polarized (CP) and linearly polarized (LP) fields with regard to the laser driven electron acceleration in vacuum and found that the $\mathrm{CP}$ field can give rise to greater acceleration efficiency.

\subsubsection{Acceleration using microwaves}

The researchers have made efforts to use microwave field for the particle acceleration [35, 36,75 - 85]. In microwave plasma interaction experiments, electron acceleration has been realized via the $\vec{v}_{p} \times \vec{B}$ process $[75,76]$ and that of resonance absorption during wave particle interaction [77]. In the $\vec{v}_{p} \times \vec{B}$ process, where $\vec{v}_{p}$ is the phase velocity of the wave, an electrostatic wave (e.g. electron plasma wave) propagates in a direction perpendicular to a magnetic field $\vec{B}$. Here an electron that is trapped in the wave trough gets accelerated in the $\vec{v}_{p} \times \vec{B}$ direction. In these experiments, the electrons could be accelerated up to 400 $\mathrm{eV}$. In another experiment, a nonlinear large amplitude ion wave was excited by using short microwave pulses at the resonance absorption region [36], where a strong electron wave was found to be excited after shut-off of the incident microwave pulse and high energy electrons got emitted and accelerated by the electron wave wakefield. Hirshfield et al. [80] have proposed a cyclotron autoresonance accelerator using rf gyroresonant acceleration, where the resonance for a TE11 mode was maintained along a waveguide by the applied magnetic field and group velocity axial tapers, and the maximum energy achieved by the electron beam in this process was up to $2.82 \mathrm{MeV}$. Yoder et al. [86] have measured the energy gain from a microwave inverse free electron laser accelerator including the energy change as a function of relative injection phase of the electron bunches. In this accelerator, the effective accelerating gradient was achieved as $0.43 \mathrm{MV} / \mathrm{m}$ and the gain for a $6 \mathrm{MeV}$ electron bunch was observed about $360 \mathrm{keV}$. Carlsten [81] has done modal analysis and gain calculation for a sheet electron beam in a ridged waveguide slow wave structure. Kumar and Malik [87] have discussed the importance of obliquely applied magnetic field to an electron acceleration and obtained that the larger acceleration is possible when the condition $\omega_{p e}>\omega_{c}\left(\omega_{p e}\right.$ is the electron plasma frequency and $\omega_{c}$ is the electron cyclotron frequency) is achieved in the plasma filled waveguide. Also it was proposed to use the field of superposed mode in waveguide for the effective electron acceleration [88].

\section{Case study: Wakefield by lasers and microwaves}

Here we take an example of wakefield excitation by short pulse lasers in an infinite plasma [38] and by the microwave pulses in a rectangular waveguide $[89,90]$.

\subsection{Wakefield by different types of laser pulses}

A laser pulse with frequency $\omega(=2 \pi f)$, intensity $I_{0}$ (corresponding field $E_{0}$ ) and pulse duration $\tau\left(=f_{p} \neg=2 \pi / \omega_{p e}\right)$ is considered to propagate in a homogeneous plasma of density $n_{0}$ 
and excite wakefield $E_{x}$ (corresponding potential $\phi$ ) behind it. The ions (density $n_{i}$ ) in the plasma are taken to be immobile on the time scale of the interest and the plasma response to the electromagnetic field is given by the following cold and collisionless electron fluid equations

$$
\begin{gathered}
\partial n_{e} / \partial t+\vec{\nabla} \cdot\left(n_{e} \vec{v}\right)=0 \\
d \vec{p} / d t=-e(\vec{E}+\vec{v} \times \vec{B}) \\
\vec{\nabla} \times \vec{E}=-\partial \vec{B} / \partial t \\
\vec{\nabla} \times \vec{B}=\mu_{0} \vec{j}+\left(1 / c^{2}\right) \partial \vec{E} / \partial t \text { with } \vec{j}=-n_{e} e \vec{v} \\
\vec{\nabla} \cdot \vec{B}=0 \\
\varepsilon_{0} \vec{\nabla} \cdot \vec{E}=e\left(n_{i}-n_{e}\right)
\end{gathered}
$$

With the help of above equations one can easily obtain the following dispersion relation for the laser propagation in the plasma $\omega^{2}=c^{2} k^{2}+\omega_{p e}^{2}$, from which the group velocity of the laser is found as $v_{g}=c \sqrt{\left(1-\omega_{p e}^{2} / \omega^{2}\right)}$. Clearly the group velocity depends on the plasma density and it can be adjusted as per the requirement.

We consider one-dimensional weakly relativistic case for the nonevolving system, i.e. when all the quantities depend only on $\xi=x-v_{g} t$, and take the electron density $n_{e}=n_{0}+n_{e}^{\prime}$ together with $n_{e}^{\prime}$ as the density perturbation due to the laser pulse and $n_{0}$ as the unperturbed density in a homogeneous plasma where $\partial n_{0} / \partial \xi=0$. Then the fluid equations are integrated under the condition that the oscillating quantities vanish as $|\xi| \rightarrow \infty$ and also when the perturbations are not so great $\left(n_{e}^{\prime} / n_{0}<<1\right)$. This yields

$$
\frac{\partial^{2} \phi}{\partial \xi^{2}}+\left(\frac{\omega_{p e}}{v_{g}}\right)^{2} \phi-\left(\frac{c^{2} e}{2 m_{e} v_{g}^{4}}\right) E^{2}=0
$$

This is the general equation for the wake potential $\phi$ which can directly use different envelopes of $E$, i.e. different shapes of the laser pulses. Here we concentrate on three types of the shapes, namely Gaussian-like (GL) pulse, rectangular-triangular (RT) pulse and rectangular-Gaussian (RG) pulse, as shown in Fig.6. 


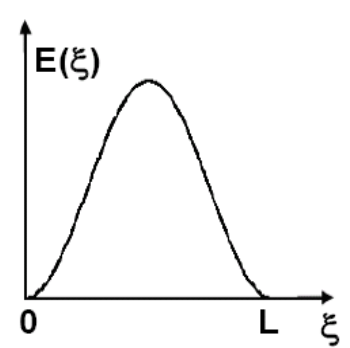

(a) GL pulse

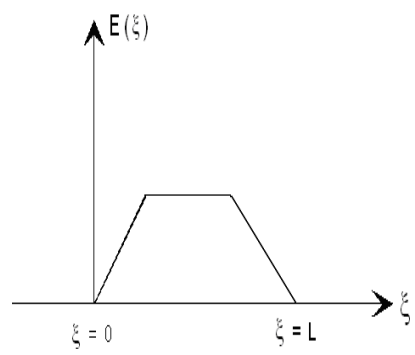

(b) RT pulse

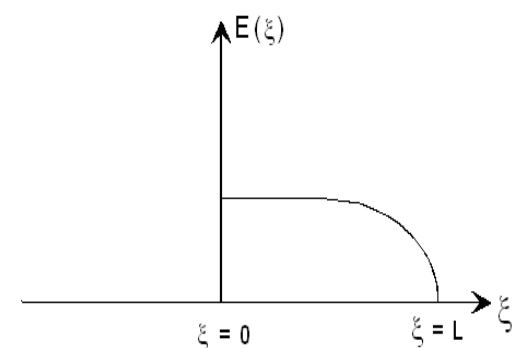

(c) RG pulse

Figure 6. Different shapes of the laser pulses with pulse length L (duration $\tau$ ).

The wakefield $E_{G L}$ for the case of GL pulse is obtained as $E_{G L}(\xi)=a_{1} \cos \left(k_{p} L / 2\right) \cos k_{p}(\xi-L / 2)$, where $a_{1}=\left(e c^{2} E_{0}^{2} \pi L / m_{e} v_{g}^{4}\right)\left[1 /\left(L^{2} \alpha^{2}-\pi^{2}\right)\right]$ and $k_{p}=$ $\omega_{p e} / v_{g}=2 \pi / \lambda_{p}$ together with $\lambda_{p}$ as the plasma wavelength, which is described by the group velocity of the laser pulse in the plasma. The density perturbations behind the pulse are obtained as $\left(n_{e}^{\prime} / n_{0}\right)_{G L}=\left(e a_{1} / 2 m_{e} v_{g}^{2} \alpha\right)\left\{2 \cos \left(k_{p} L / 2\right) \sin k_{p}(\xi-L / 2)+\left(L^{2} \alpha^{2}-\pi^{2}\right) / \alpha \pi L\right\}$.

The wakefield $E_{R T}$ for the case of RT pulse is obtained as $E_{R T}(\xi)=a_{2} a_{3} \cos k_{p}(\xi-L / 2)$, where $a_{2}=\left(e c^{2} E_{0}^{2} / 2 m_{e} v_{g}^{4} \alpha\right)$ and

$$
a_{3}=\left\{\begin{array}{l}
{\left[c_{1}-1-\left(\frac{2 \pi c_{1}}{L \alpha}\right) \sin \left(k_{p} L / 4\right)+\frac{\left(1-c_{2}\right)}{2} \cos \left(k_{p} L\right)+\frac{\left(1-c_{2}\right)}{2} \cos \left(2 k_{p} L / 3\right)\right] \sin \left(k_{p} L / 2\right)} \\
-\left[\frac{2 \pi c_{1}}{L \alpha} \cos \left(k_{p} L / 4\right)+\frac{\left(1-c_{2}\right)}{2} \sin \left(k_{p} L\right)+\frac{\left(1-c_{2}\right)}{2} \sin \left(2 k_{p} L / 3\right)\right] \cos \left(k_{p} L / 2\right)
\end{array}\right\}
$$

together with $c_{1}=L^{2} \alpha^{2} /\left(L^{2} \alpha^{2}-4 \pi^{2}\right)$ and $c_{2}=L^{2} \alpha^{2} /\left(L^{2} \alpha^{2}-9 \pi^{2}\right)$. The density perturbations behind the pulse are obtained as $\left(n_{e}^{\prime} / n_{0}\right)_{R T}=\left(e a_{2} / m_{e} v_{g}^{2} \alpha\right)\left[1+a_{3} \sin k_{p}(\xi-L / 2)\right]$.

For the case of RG pulse the wakefield $E_{R G}$ and density perturbations $\left(n_{e}^{\prime} / n_{0}\right)_{R G}$ are calculated as $E_{R G}(\xi)=-a_{4} a_{5} \cos k_{p}(\xi-L / 2)$, $\left(n_{e}^{\prime} / n_{0}\right)_{R G}=-\left(e a_{4} a_{5} / m_{e} v_{g}^{2} \alpha\right)\left\{a_{5} \sin k_{p}(\xi-L / 2)-\left(L^{2} \alpha^{2}-\pi^{2}\right)\right\}$. The constants $a_{4}$ and $a_{5}$ in these expressions are given by

$$
\begin{aligned}
& a_{4}=e c^{2} E_{0}^{2} /\left\{2 m_{e} v_{g}^{4} \alpha\left(L^{2} \alpha^{2}-\pi^{2}\right)\right\} \\
& a_{5}=\left[\begin{array}{l}
\left.\left\{2\left(L^{2} \alpha^{2}-\pi^{2}\right) \sin ^{2}\left(k_{p} L / 4\right)+L^{2} \alpha^{2} \cos \left(k_{p} L / 2\right)-\pi \alpha L \sin \left(k_{p} L\right)\right\} \sin \left(k_{p} L / 2\right)\right] . \\
-\left\{\pi^{2} \sin \left(k_{p} L / 2\right)+\pi \alpha L \cos \left(k_{p} L\right)\right\} \cos \left(k_{p} L / 2\right)
\end{array}\right]
\end{aligned}
$$




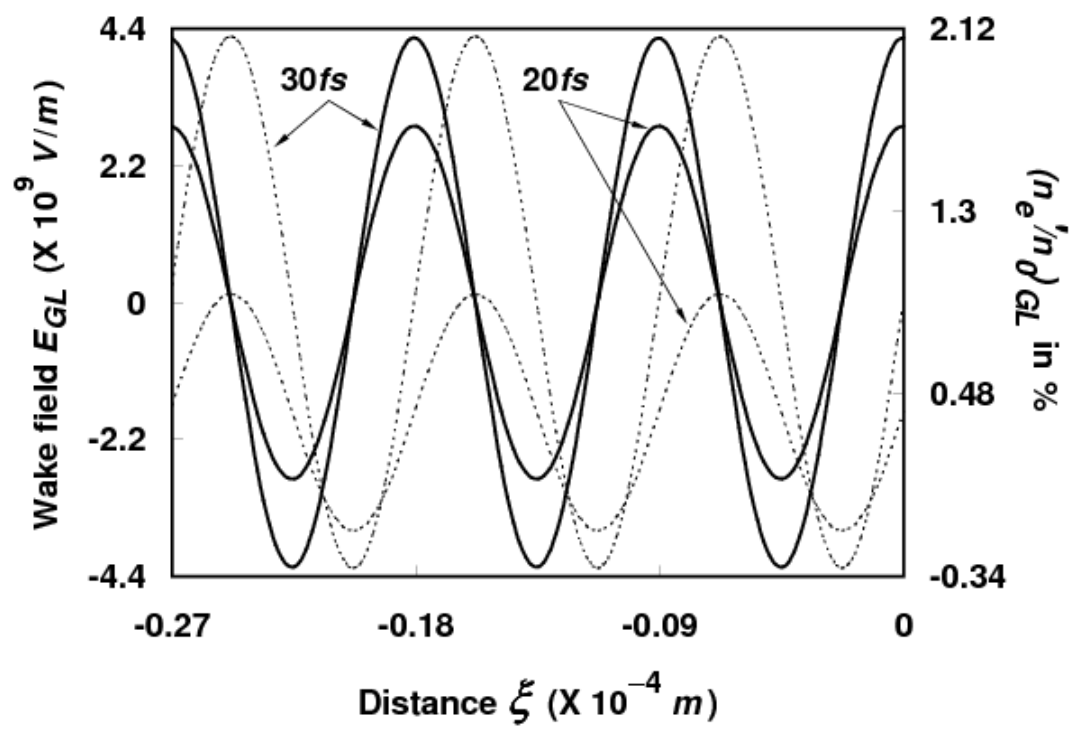

Figure 7. Wakefield $E_{G L}$ and density perturbations $\left(n_{e}^{\prime} / n_{0}\right)_{G L}$ behind the Gaussian-like pulse up to distance $3 L$ for different pulse durations $\left(\tau=20 \mathrm{ps}, 30 \mathrm{ps}\right.$ ) when the laser intensity is $3 \times 10^{18} \mathrm{~W} / \mathrm{m}^{2}$ and laser frequency is $1.6 \mathrm{PHz}$.

In Fig. 7 we show the variation of wakefield $E_{G L}$ by solid line graphs and of density perturbations $\left(n_{e}^{\prime} / n_{0}\right)_{G L}$ by dotted line graphs behind the laser pulse $(\xi<0)$ up to the distance $\xi=3 L$ when $f=1.6 \mathrm{PHz}$ and $I_{0}=3 \times 10^{18} \mathrm{~W} / \mathrm{m}^{2}$. The wakefield of the strength of $4.24 \times 10^{9} \mathrm{~V} / \mathrm{m}$ is excited by the GL pulse and the density perturbations $n_{e}{ }^{\prime}=0.0208$ times of the unperturbed density $n_{0}\left(=1.37 \times 10^{25} / \mathrm{m}^{3}\right)$ for the pulse duration of $30 \mathrm{fs}$. A comparison of the graphs marked with $30 \mathrm{fs}$ and $20 \mathrm{fs}$ reveals that the effect of pulse duration is to increase the wakefield as well as the density perturbations. This may be attributed to the decreased plasma density $n_{0}$ for the larger pulse duration as we considered $\tau=f_{p e}-1=2 \pi /$ ape as a condition for the wakefield excitation. For the fixed laser intensity, larger perturbations are realized in relatively lower density plasma and hence the enhanced field is obtained. Similar effects are observed for the cases of RT (Fig.8) and RG (Fig.9) pulses. Here the wakefield of the strength of $4.98 \times 10^{9} \mathrm{~V} / \mathrm{m}\left(4.28 \times 10^{9} \mathrm{~V} / \mathrm{m}\right)$ and density perturbations of $0.023(0.0209)$ times of the unperturbed density are obtained in case of RT (RG) pulse of the same duration of 30 $f$ s. This can also be seen that the pulses of higher intensity produce relatively larger wakefields and the density perturbations. However, a very weak effect of the laser frequency is noticed on the wakefields. In the present study of three pulses infers that the rectangular-triangular (RT) pulse is more suitable for the purpose of wakefield excitation in a homogeneous plasma. 


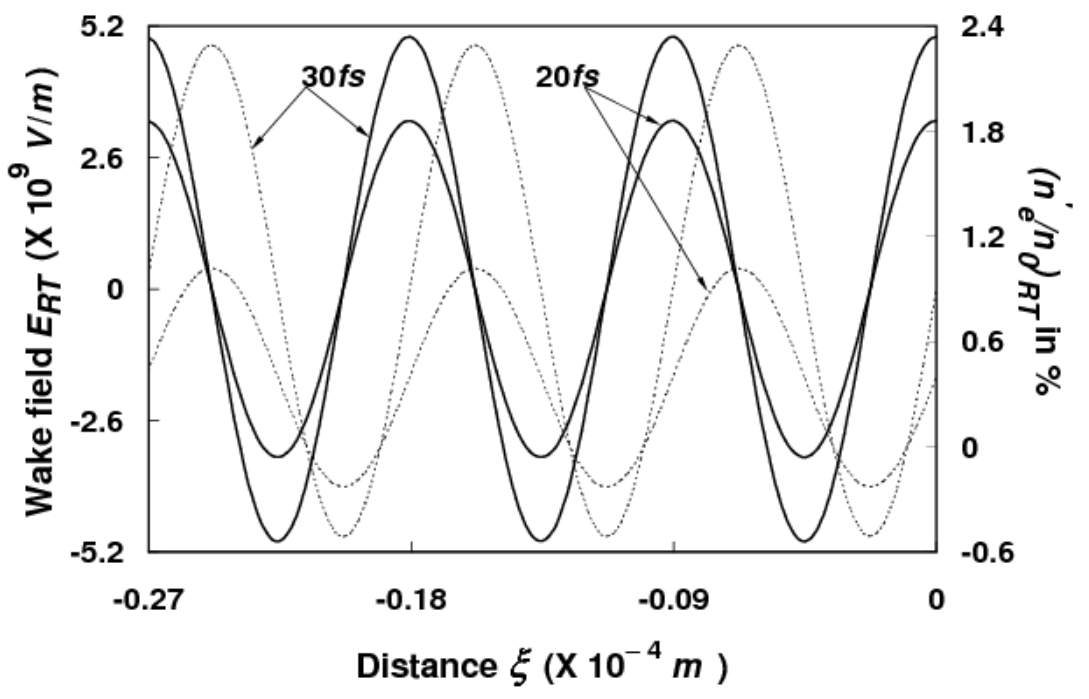

Figure 8. Wakefield $E_{R T}$ and density perturbations $\left(n_{e}^{\prime} / n 0\right)_{R T}$ behind the rectangular-triangular pulse up to distance $3 L$ for different pulse durations ( $\tau=20 \mathrm{ps}, 30 \mathrm{ps}$ ) and other parameters the same as in Fig.7.

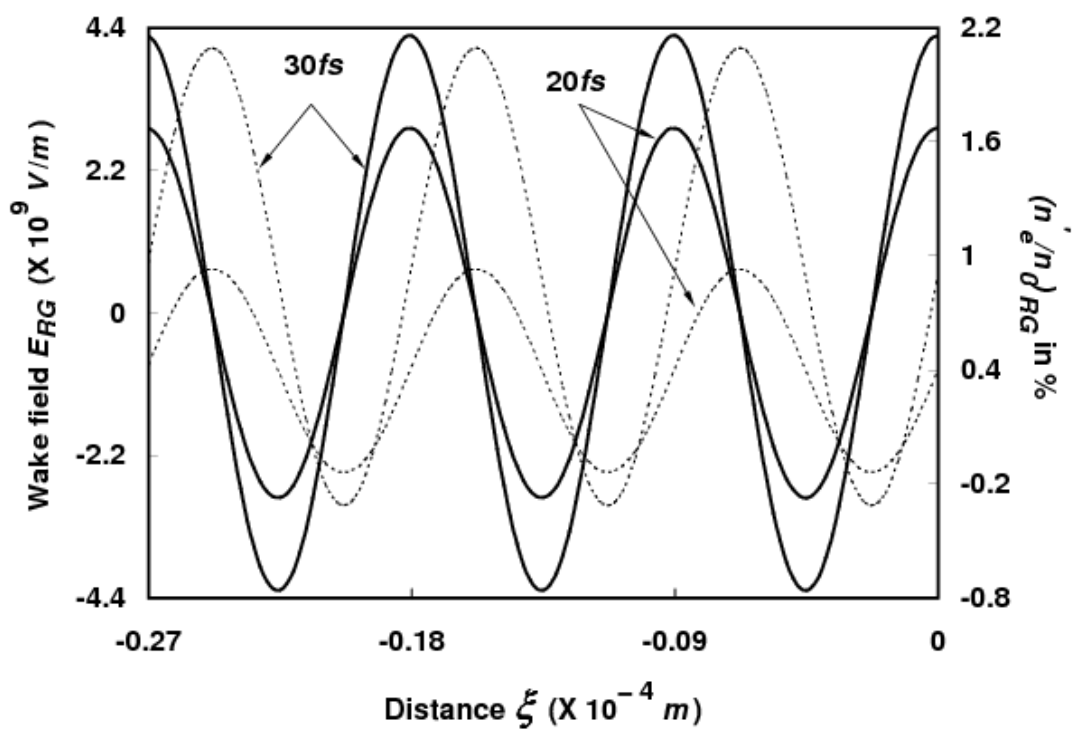

Figure 9. Wakefield $E_{R G}$ and density perturbation $\left(n_{e}^{\prime} / n_{0}\right)_{R G}$ behind the rectangular-Gaussian pulse up to distance $3 L$ for different pulse durations ( $\tau=20 \mathrm{ps}, 30 \mathrm{ps}$ ) and other parameters the same as in Fig.7. 


\subsubsection{Calculation of electron energy gain}

In order to calculate the energy gain achieved by the electron, we proceed with the momentum equation $\frac{d p}{d t}=-e E(\xi)$ and the relativistic factor $\gamma$ relation $\gamma=\sqrt{1+p^{2} / m_{e}{ }^{2} c^{2}}$.

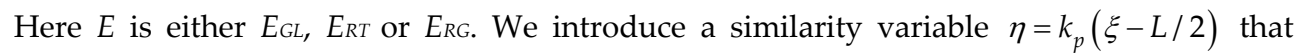
represents the phase of the wakefield as seen by the electron. For one-dimensional motion of the electron and $\xi=x-v_{g} t$, we obtain $\frac{d \gamma}{d t}=-\frac{p e E(\eta)}{\gamma m_{e}{ }^{2} c^{2}}$ and $\frac{d \eta}{d t}=k_{p}\left[c\left(1-1 / \gamma^{2}\right)^{\frac{1}{2}}-v_{g}\right]$ from the above relations. Dividing $d \gamma / d t$ by $d \eta / d t$ and integrating the resultant equation by taking $d x / d t=v_{x}=c\left(1-1 / \gamma^{2}\right)^{1 / 2}$ and $\beta_{r}=v_{g} / c$ we get

$$
\gamma-\beta_{r}\left(\gamma^{2}-1\right)^{\frac{1}{2}}=-\frac{e}{m_{e} c^{2} k_{p}} \int E(\eta) d \eta
$$

This is the general equation that describes the electron acceleration in the wakefield $E(\eta)$. By using the expressions of wakefield $E$ for different shapes of the laser pulses we can determine the corresponding relativistic factor (or the energy gain).

For the case of GL pulse, the integration of the resultant equation with the initial value of $\gamma$ as $\gamma 0$ at $\xi=0$ yields

$$
\gamma-\gamma_{0}-\beta_{r}\left[\left(\gamma^{2}-1\right)^{\frac{1}{2}}-\left(\gamma_{0}^{2}-1\right)^{\frac{1}{2}}\right]=-\frac{e^{2} E_{0}^{2} \pi L}{m_{e}^{2} v_{g}^{4} k_{p}\left(L^{2} \alpha^{2}-\pi^{2}\right)} \cos \left(k_{p} L / 2\right)\left\{\sin \eta+\sin \left(\frac{k_{p} L}{2}\right)\right\} .
$$

Without loss of generality we can assume $\gamma^{2}, \gamma_{0}^{2} \gg>1$. Hence, $\Delta \gamma_{G L}=\gamma-\gamma_{0}=-\frac{e^{2} E_{0}^{2} \pi L}{m_{e}^{2} v_{g}^{4} k_{p}\left(1-\beta_{r}\right)\left[L^{2} \alpha^{2}-\pi^{2}\right]} \cos \left(\frac{k_{p} L}{2}\right)\left\{\sin \eta+\sin \left(\frac{k_{p} L}{2}\right)\right\} . \quad$ Therefore, the energy gain obtained by the electron during its acceleration in the wakefield excited by the GL pulse can be given by $\Delta W_{G L}=m_{e} c^{2} \Delta \gamma_{G L}$

Similarly the electron energy gain in the case of RT and RG pulses are obtained as

$$
\Delta W_{R T}=-\frac{e a_{2} a_{3}}{k_{p}\left(1-\beta_{r}\right)}\left\{\sin \eta+\sin \left(\frac{k_{p} L}{2}\right)\right\} \text { and } \Delta W_{R G}=\frac{e a_{4} a_{5}}{k_{p}\left(1-\beta_{r}\right)}\left\{\sin \eta+\sin \left(\frac{k_{p} L}{2}\right)\right\} .
$$

Now we examine the effects of pulse duration (plasma density), laser intensity and laser frequency on the electron acceleration for different shapes of the laser pulses and make a comparative study. 


\subsubsection{Effect of pulse duration $\tau$}

We have already seen that the wakefield gets enhanced with the increased pulse duration $\tau$ for all the shapes of the laser pulses. Therefore, it is obvious that the electron will gain larger energy in the wakefield, which is excited by the pulses of longer durations. The same has been portrayed in Fig.10 for the laser intensity of $3 \times 10^{18} \mathrm{~W} / \mathrm{m}^{2}$ and its frequency as $1.6 \mathrm{PHz}$. A comparison of the three graphs infers that the energy gains follow the trend $W_{R T}>W_{R G}>W_{G L}$. The increased gain for the longer pulse durations is attributed to the enhanced plasma wavelength $\lambda_{p}$. Since $\lambda_{p}$ is independent of the pulse shapes, the electron gets larger energy for the increasing $\tau$ for all types of the pulses irrespective of their shapes. This can also be seen from this figure that the change in energy gain is faster when the pulses of longer durations are employed for the wakefield excitation.

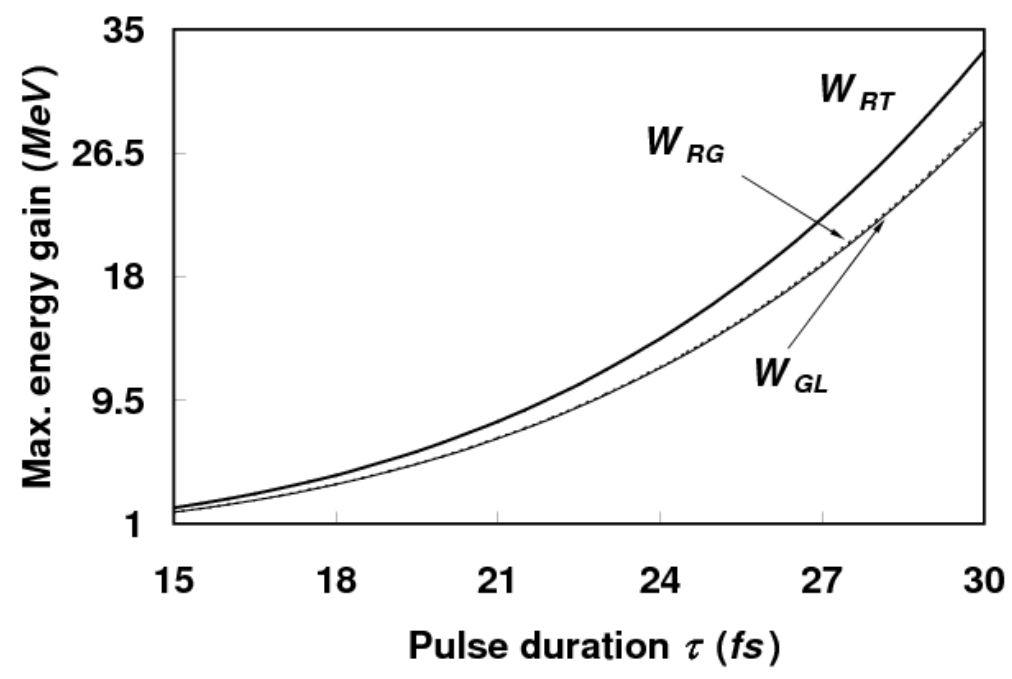

Figure 10. Dependence of maximum energy gain of electron on the laser pulse duration for the same parameters as in Fig.2. $W_{R T}$ is the gain in case of rectangular-triangular pulse, $W_{R G}$ is for rectangularGaussian pulse and $W_{G L}$ is for Gaussian-like pulse.

\subsubsection{Effect of laser frequency $f$}

The effect of laser frequency $f$ on the maximum energy gain attained by an electron is shown in Fig.11, from where it is evident that the gain is larger in case of RT pulse. Moreover, the slopes of the graphs reveal that the effect of laser frequency is more significant in the case of RT pulse in comparison with RG and GL pulses. Since the wakefield and plasma wavelength show weak dependence on the frequency $f$, it is worth clarifying the main factor that leads to significant increase in the electron energy gain with $f$. Actually a slight change in $v_{g}$ due to $f$ causes a greater change in the factor $\left(1-\beta_{r}\right)$ appearing in the denominator of the energy gain expressions. Since $v_{g}$ increases for the larger frequencies, the gain gets larger with the increasing laser frequencies. 


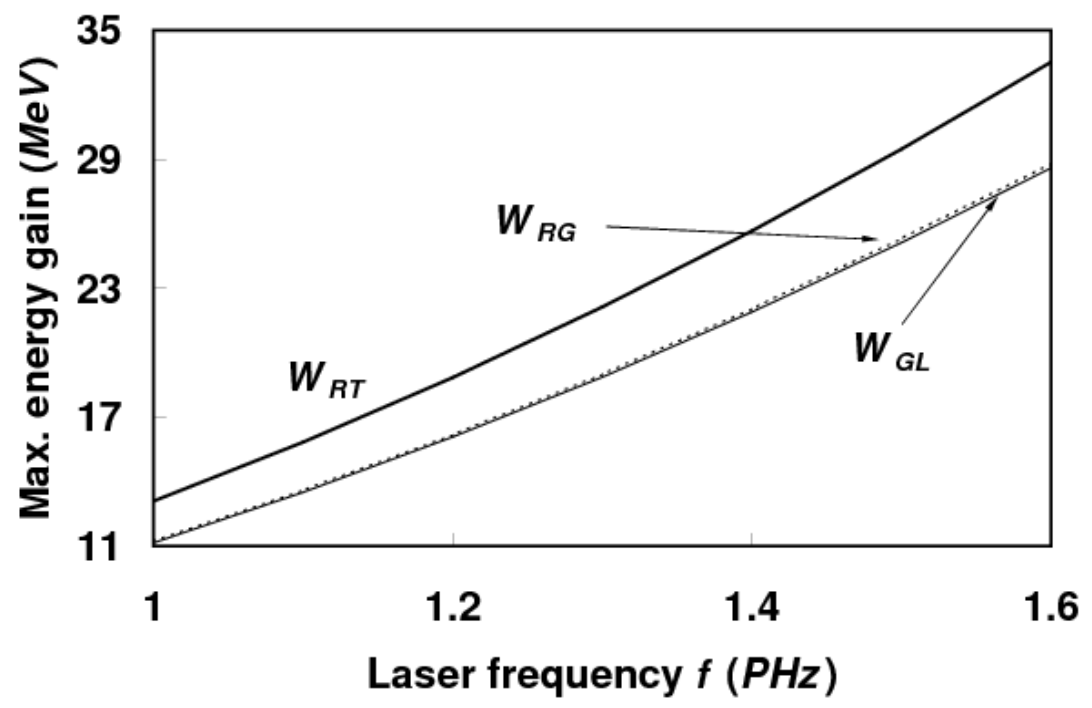

Figure 11. Dependence of maximum energy gain of electron on the laser frequency when the laser intensity is $3 \times 10^{18} \mathrm{~W} / \mathrm{m}^{2}$ and pulse duration is $30 \mathrm{fs}$. $W_{R T}, W_{R G}$ and $W_{G L}$ have the same meaning as in Fig.10.

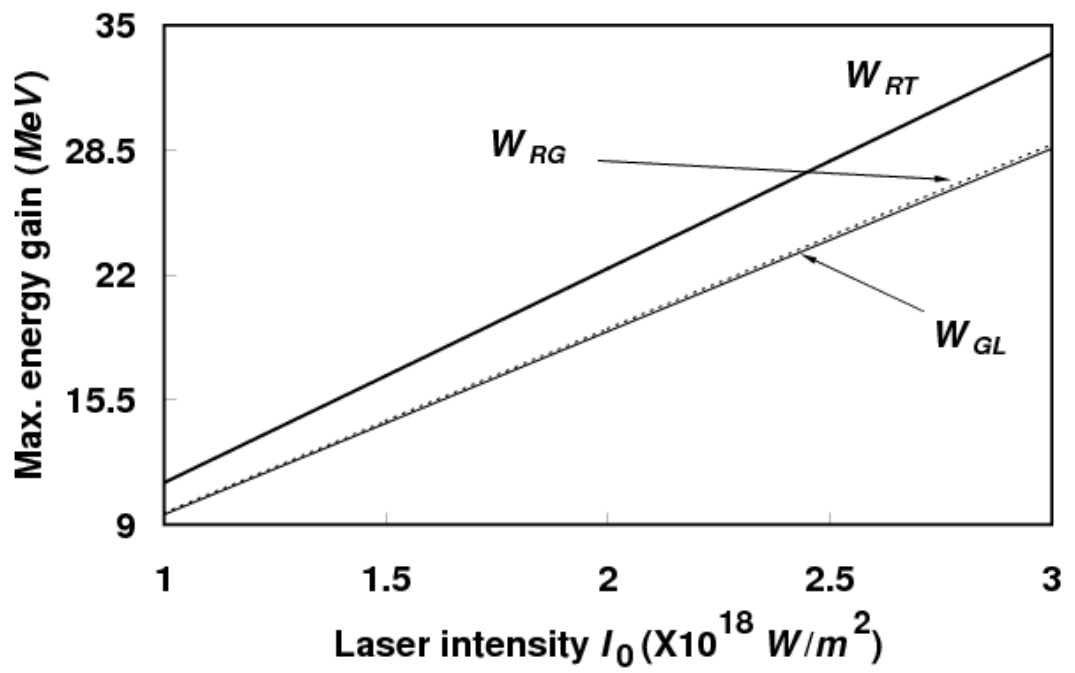

Figure 12. Variation of maximum energy gain of electron with the laser intensity when the pulse duration is $30 \mathrm{fs}$ and laser frequency is $1.6 \mathrm{PHz}$. $W_{R T}, W_{R G}$ and $W_{G L}$ have the same meaning as in Fig.10. 


\subsubsection{Effect of laser intensity Io}

The expressions of wakefield for all types of the pulses show that the wake amplitude is directly proportional to $E_{0}^{2}$, i.e. the intensity of the laser. It means higher intensity pulses will excite larger amplitude wakefield owing to the larger density perturbations in the plasma. However, we cannot indefinitely increase the amplitude of the wakefield because there is a limit on the maximum field that a plasma can support. Figure 12 shows that the maximum energy gain is increased from $9.5 \mathrm{MeV}$ to $33.5 \mathrm{MeV}$, when the laser intensity is raised from $1 \times 10^{18} \mathrm{~W} / \mathrm{m}^{2}$ to $3 \times 10^{18} \mathrm{~W} / \mathrm{m}^{2}$ in case of RT pulse. A comparison of all the graphs shows that the RT pulse supersedes and gives the best results. Also the difference in energy gain becomes more and more significant when the intensity of the pulses is increased. The better results, i.e. higher amplitude wakefield and larger energy gain, obtained in case of RT pulse having smooth/fast rising time are consistent with the observations of Bulanov et al. [91] where he observed regular wakefields by a pulse with sharp steepening of its leading front.

\subsection{Wakefield by different microwave pulses in waveguides}

Here we present some results on wakefield excitation in a waveguide by different shapes of the microwave pulses, i.e. GL, RG and RT pulses.

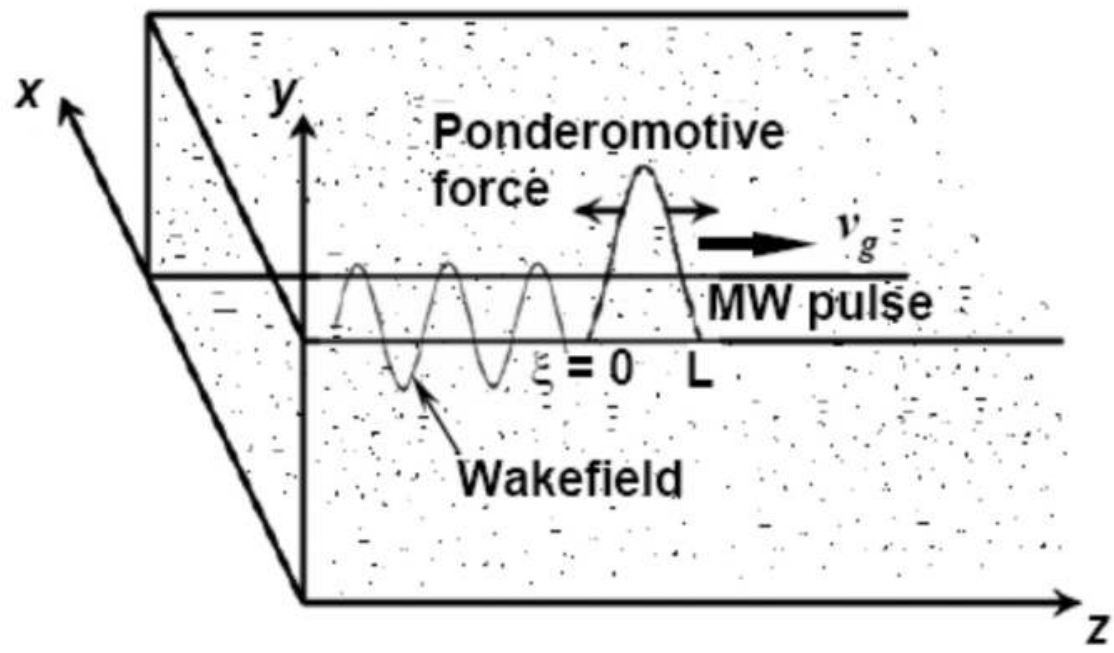

Figure 13. Schematic of wakefield generation in plasma filled rectangular waveguide by microwave pulse. Here $\xi=z-v_{g} t$ and $L$ is the pulse width.

We consider that a microwave pulse of pulse duration $\tau$ at a frequency $f$ propagates in a plasma filled $\mathrm{b} \times h$ rectangular waveguide. This pulse resonantly excites the wakefield (corresponding potential $\phi$ ) in the waveguide under the action of ponderomotive force (Fig.13), when the pulse duration $\tau$ matches with the inverse of the plasma frequency, i.e. 
$\tau=f_{p e}{ }^{-1}$. The electric and magnetic fields associated with the microwave are represented by $\vec{E}$ and $\vec{B}$. We use the Maxwell's equations and obtain the group velocity of the microwave pulse as $v_{g}=c\left(1-\omega_{p}^{2} / \omega^{2}-\pi^{2} c^{2} / b^{2} \omega^{2}\right)^{1 / 2}$ that coincides with the phase velocity of the wakefield.

For the rectangular waveguide, we take the distribution of the microwave field as $\vec{E}=\hat{y} E(\xi) \sin \left(\frac{\pi x}{b}\right)$ and $\vec{B}=\hat{x} B_{x}(\xi) \sin \left(\frac{\pi x}{b}\right)+\hat{z} B_{z}(\xi) \cos \left(\frac{\pi x}{b}\right)$. Using these relations in the basic fluid equations, we integrate them under the conditions that all the oscillating quantities tend to zero as $\xi \rightarrow \infty$ under the weakly nonlinear theory. With this we get the following equation

$$
\frac{\partial^{2} \phi}{\partial \xi^{2}}+\frac{e}{2 m_{e} v_{g}^{2}}\left(\frac{\partial \phi}{\partial \xi}\right)^{2}+\frac{\omega_{p}^{2}}{v_{g}^{2}} \phi-\left(\frac{c^{2} e}{m_{e} v_{g}^{2}}\right) \sin \left(\frac{\pi x}{b}\right) F(\xi)=0,
$$

where

$$
F(\xi)=\frac{1}{2}\left[\left(\frac{1}{v_{g}^{2}}-\frac{1}{c^{2}}\right) E^{2}(\xi)-B_{z}^{2}(\xi)\right] .
$$

This equation can be viewed as the equation governing the forced (driven) harmonic oscillator. Here the last term is the force term that evolves due to the microwave field (ponderomotive force) and drives the wake in the plasma. The third term is proportional to $\phi$ and hence its coefficient determines the natural frequency of the wake. The second term is the damping term through which the nonlinearity enters the system as it is proportional to square of $\partial \phi / \partial \xi$ (nonlinear term). Thus, the wake with potential $\phi$ is evolved in the plasma as a combined contribution of each term of Eq.(42).

We can use the information related to the shape of the pulse through the last term of Eq.(42) via the coefficient $F(\xi)$. Using the fourth-order Runge-Kutta method we simulate this equation for the above-mentioned three types of the pulse shapes. Here we take different profiles for the electric field of the pulse keeping in mind its shape and calculate $B_{z}(\xi)$ with the help of Maxwell's equation. The relation between $B_{z}(\xi)$ and $E(\xi)$ thereby comes out to be $B_{z}(\xi)=\frac{1}{v_{g}} \frac{\pi}{b} \int E(\xi) d \xi$.

\subsubsection{Results on wakefield in the waveguide}

As mentioned, we solve Eq.(42) numerically and obtain the potential $\phi$ from which we look for the wakefield amplitude for the mentioned three types of the pulse shapes. Figures $14-$ 
16 show the profile of wakefield generated by GL pulse, RG pulse and RT pulse, respectively. It can be easily seen that the amplitude of the wakefield is the largest in the case of RT pulse and is the least for the case of GL pulse; in other words, the wakefield amplitude follows the trend $E_{R T}>E_{R G}>E_{G L}$.

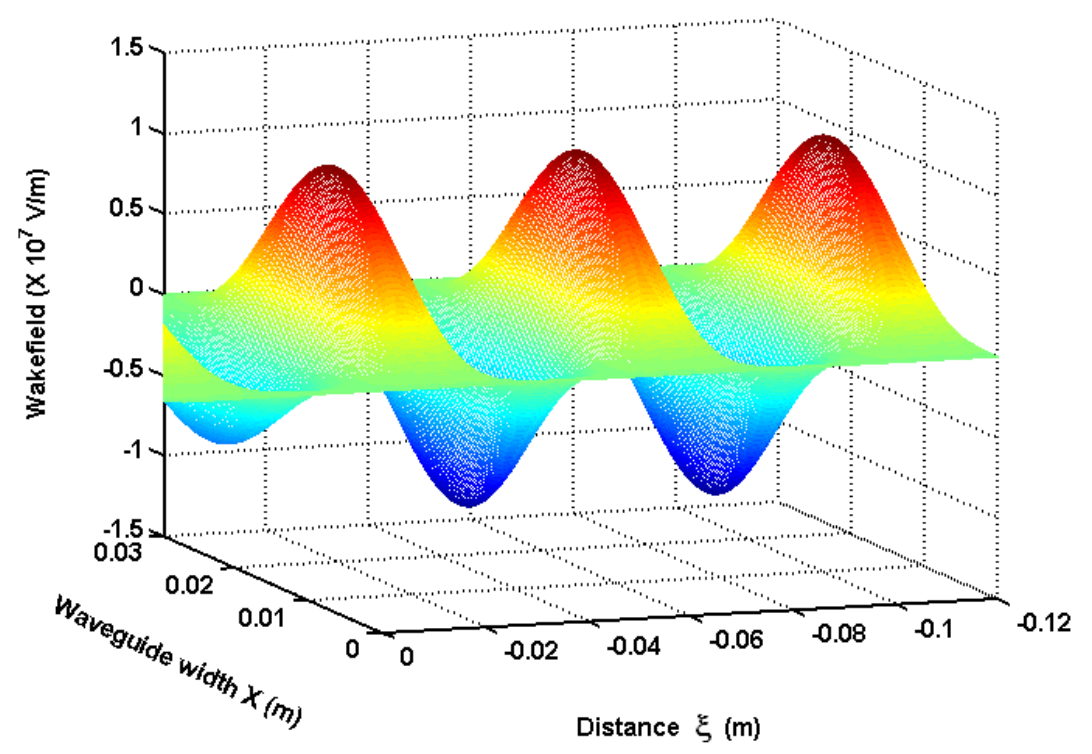

Figure 14. Variation of wakefield generated by microwave GL pulse in a waveguide for microwave intensity $I=2 \mathrm{GW} / \mathrm{m}^{2}$, frequency $f=30 \mathrm{GHz}$, pulse duration $\tau=2 n s$, plasma density $n_{0}=4.5 \times 10^{17} \mathrm{~m}^{-3}$ and waveguide width $b=0.03 \mathrm{~m}$. 


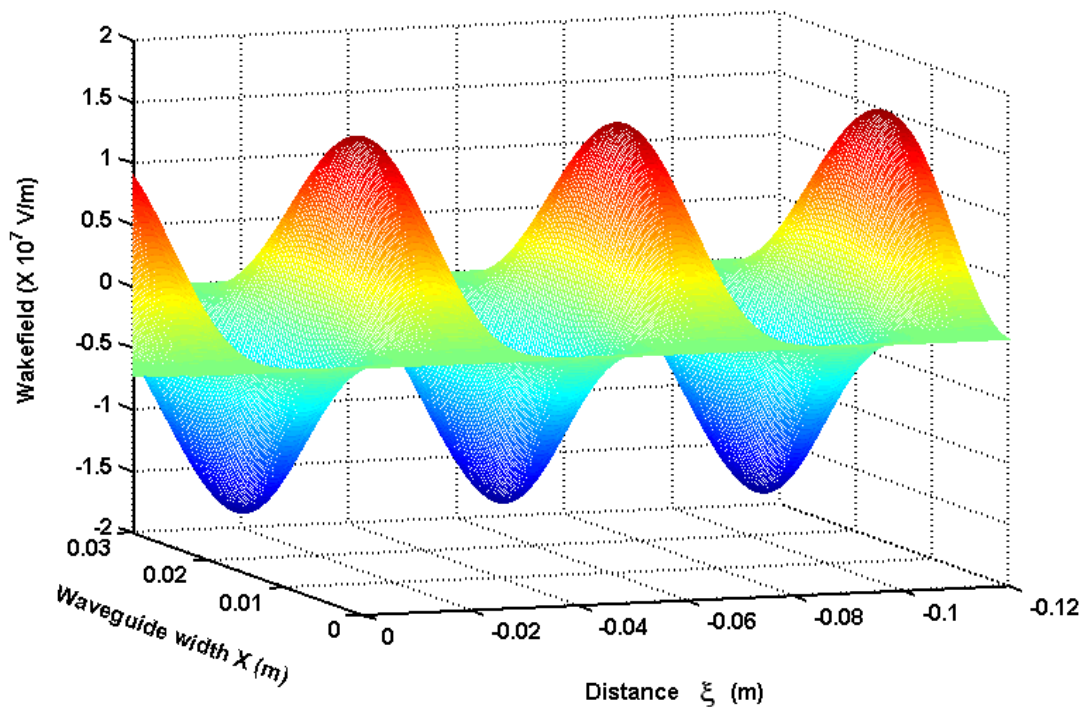

Figure 15. Variation of wakefield generated by microwave RG pulse in a waveguide for the same parameters as in Fig.14.

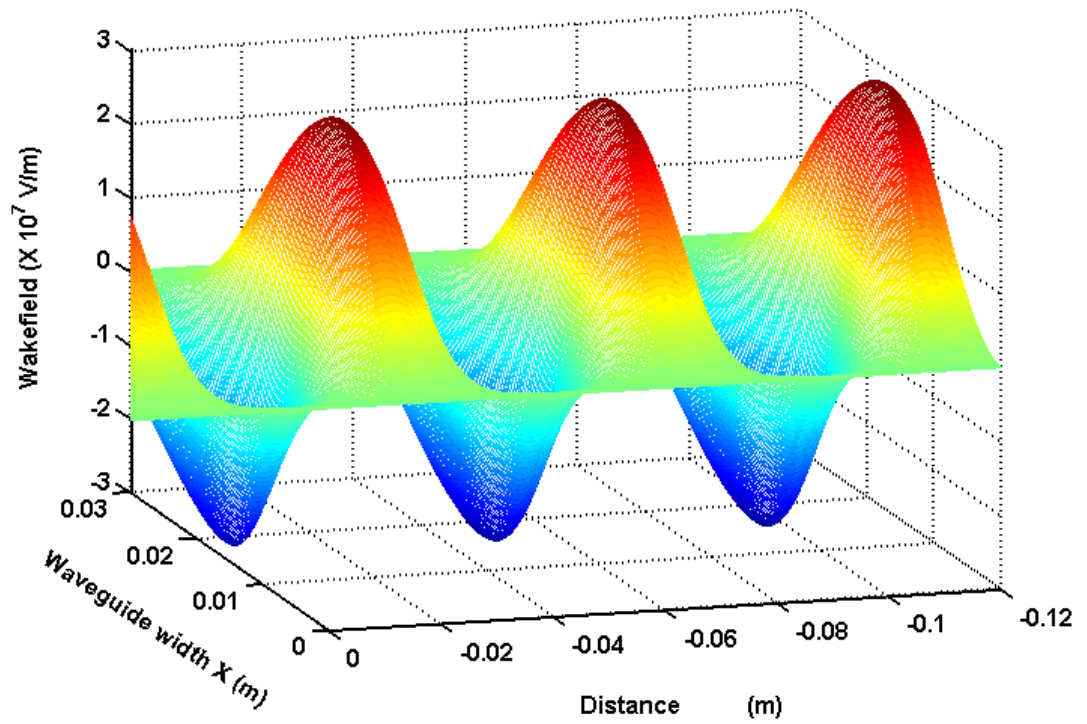

Figure 16. Variation of wakefield generated by microwave RT pulse in a waveguide for the same parameters as in Fig.14. 


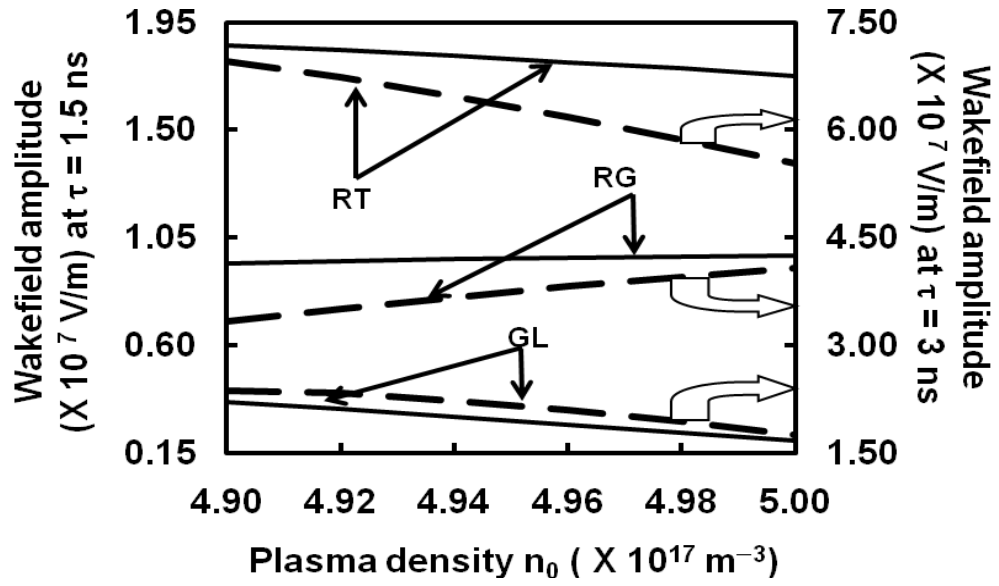

Figure 17. Dependence of wakefield amplitude on plasma density $n_{0}$ for two different microwave pulse durations $\tau=1.5 \mathrm{~ns}$ (solid line graphs, left axis) and $\tau=3.0 \mathrm{~ns}$ (dashed line graphs, right axis), when the other parameters are the same as in Fig.14.

Dependence of the wakefield amplitude on the plasma density for two different pulse durations is shown in Fig.17, from where it is found that the amplitude is increased for the higher plasma density in the waveguide for the case of RG pulse. However, the opposite trend is realized for the other types of the pulses (GL and RT pulses); the wakefield amplitude remains the largest in case of the RT pulse. A comparison of slopes of the graphs yields that the RT pulse shows stronger dependence on the plasma density in comparison with the other types of the pulses. With regard to the effect of pulse duration, we notice that the larger wakefield is obtained for the case of longer pulse durations; this is true for all types of the pulses.

In Fig.18, the effects of microwave frequency and its intensity are studied on the wakefield amplitude, where it is seen that an increase in the frequency leads to an enhancement in the wakefield amplitude for the cases of RG pulse and GL pulse; opposite is true for the RT pulse, which also shows a strong dependence (slope 0.097 at $I=2 \times 10^{9} \mathrm{~W} / \mathrm{m}^{2}$ ) on the frequency as compared with the other pulses. With regard to the effect of microwave intensity, we observe that the larger wakefield is obtained for the higher microwave intensity. This is further evident that the amplitude is modified at a faster rate in the case of RT pulse in comparison with the other pulses. Generally, we can conclude that the RT pulse is most sensitive to microwave frequency and intensity.

It is worth noticing from Figs. $14-18$ that tens of $\mathrm{MV} / \mathrm{m}$ wakefield is attained with the use of moderate intensity microwave pulses. Therefore, in view of the effect of microwave intensity (Fig.18), it is expected that the wakefield of the order of GV/m can be generated if the microwave pulses of intensity $\sim \mathrm{TW} / \mathrm{m}^{2}$ are available. Since the wakefield of this order is generally obtained by ultra high intensity lasers in usual wakefield generation schemes, the present mechanism of exciting wakefield in the waveguide by microwave pulses seems to be more effective and feasible as it can reduce the cost of accelerator and also it will provide an additional controlling parameter (the waveguide width). 


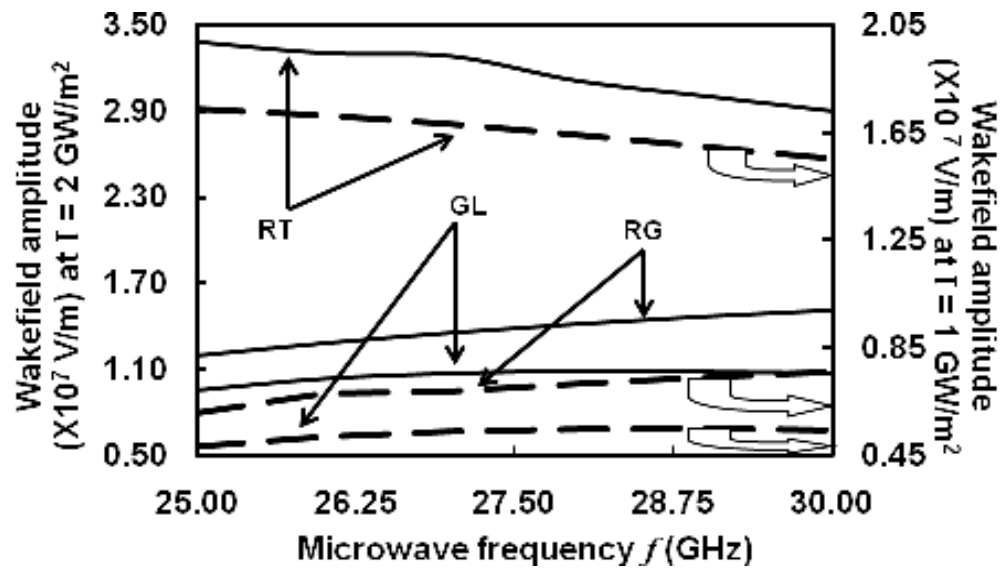

Figure 18. Variation of wakefield amplitude with microwave frequency $f$ for two different microwave intensities $I=2 \times 10^{9} \mathrm{~W} / \mathrm{m}^{2}$ (solid line graphs, left axis) and $I=1 \times 10^{9} \mathrm{~W} / \mathrm{m}^{2}$ (dashed line graphs, right axis), when the other parameters are the same as in Fig. 14.

Variation of the wakefield amplitude with the waveguide width is shown in Fig.19, where it is observed that the amplitude is decreased with the increase of waveguide width; same result was obtained in an analytical calculation [38]. It means the larger wakefield can be obtained for the case of plasma filled narrower waveguide. A comparison of the slopes of the graphs reveals that the wakefield amplitude changes at a faster rate in the case of RT pulse. Therefore, the RT pulse is found to be more sensitive to the waveguide width. Thus, we can conclude that a plasma filled narrower waveguide is best suited for an effective wakefield excitation and the significant particle acceleration if the RT pulse is used.

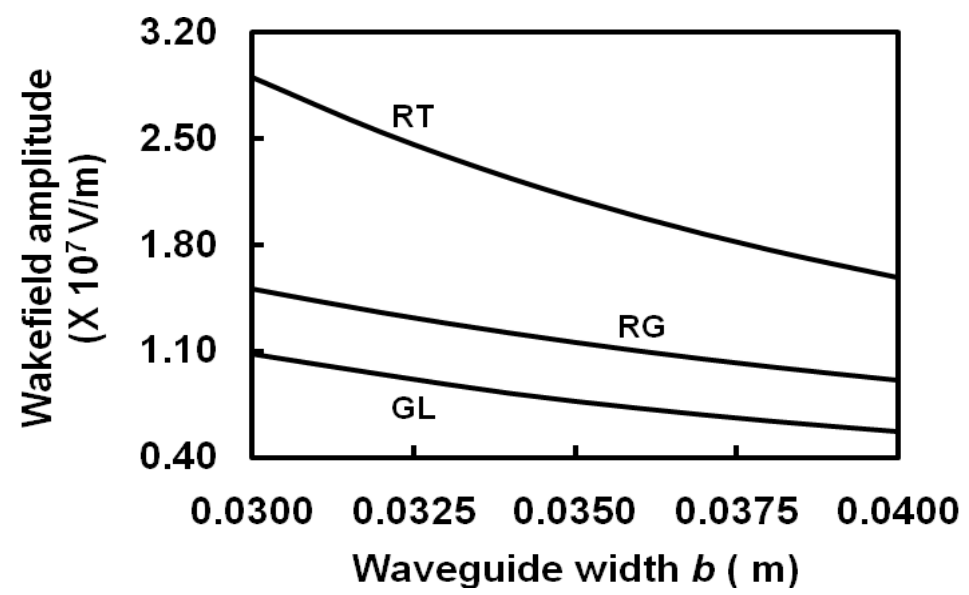

Figure 19. Variation of wakefield amplitude with waveguide width. The values of intensity, frequency, pulse duration and plasma density are given in the caption of Fig. 14. 


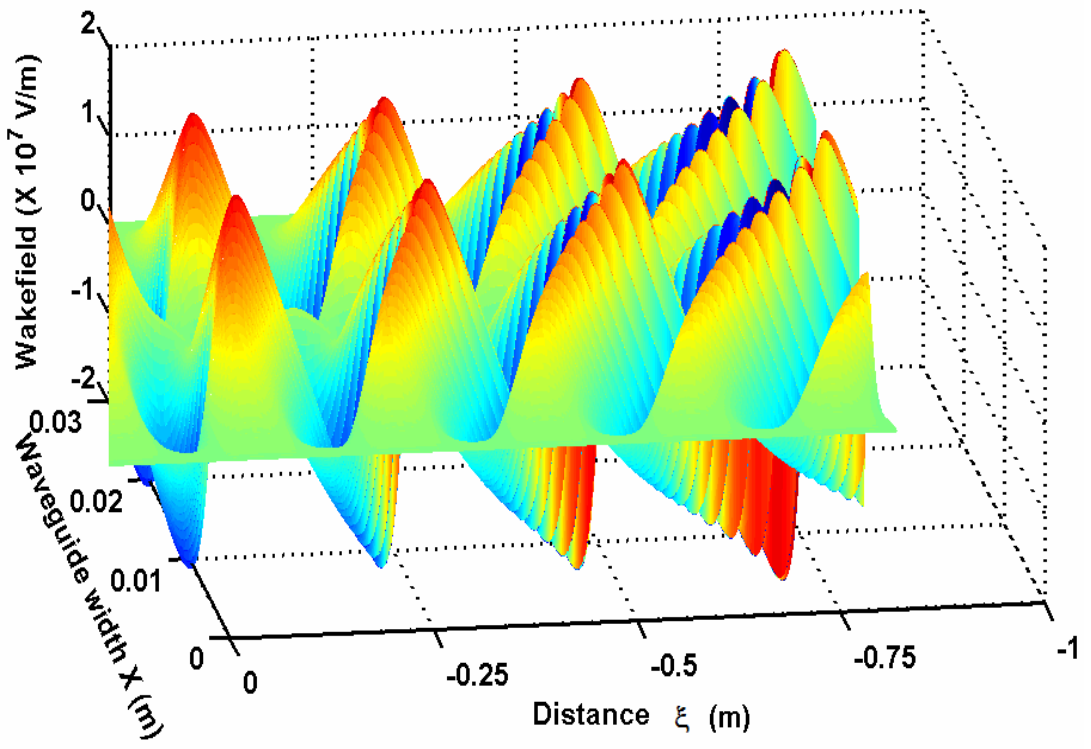

Figure 20. Profile of wakefield $E_{W}$ in a plasma filled waveguide near cutoff conditions when $f=6.76 \mathrm{GHz}, \tau=0.9 \mathrm{~ns}, f_{c}=5.11 \mathrm{GHz}, I=1 \times 10^{9} \mathrm{~W} / \mathrm{m}^{2}$ and $b=0.03 \mathrm{~m}$.

\subsubsection{Wakefield near cutoff conditions}

This has already been explored that the wakefield of larger amplitude is obtained for the smaller waveguide width and the longer pulse duration. For fixed microwave frequency, the cutoff frequency $f_{c}$ gets higher under the effect of decreased width $b$ and plasma density $n_{0}$. These effects can be viewed as if the microwave frequency is brought near the cutoff frequency $f_{c}$. Therefore, it is of much importance to investigate the wakefield structure near cutoff conditions, i.e. when $f$ is near $f_{c}$. These results are presented in Figs.20 and 21 for $f=6.76 \mathrm{GHz}$ whereas $f_{c}=5.11 \mathrm{GHz}$. Fig.20 shows that the amplitude of wake wave gets increased under this situation as we move away from the microwave pulse, i.e. for decreasing values of $\xi$. This is further noticed that various peaks develop along the waveguide width during the growth of wakefield amplitude and it becomes unstable. Thus, it is plausible that some instability develops near the cutoff conditions. In order to further investigate this effect, we show in Fig.21 the maximum distance by which this growth occurs. Here we observe that the amplitude gets terminated around $\xi=3.7 \mathrm{~L}$ in the plasma and the field breaks down. 


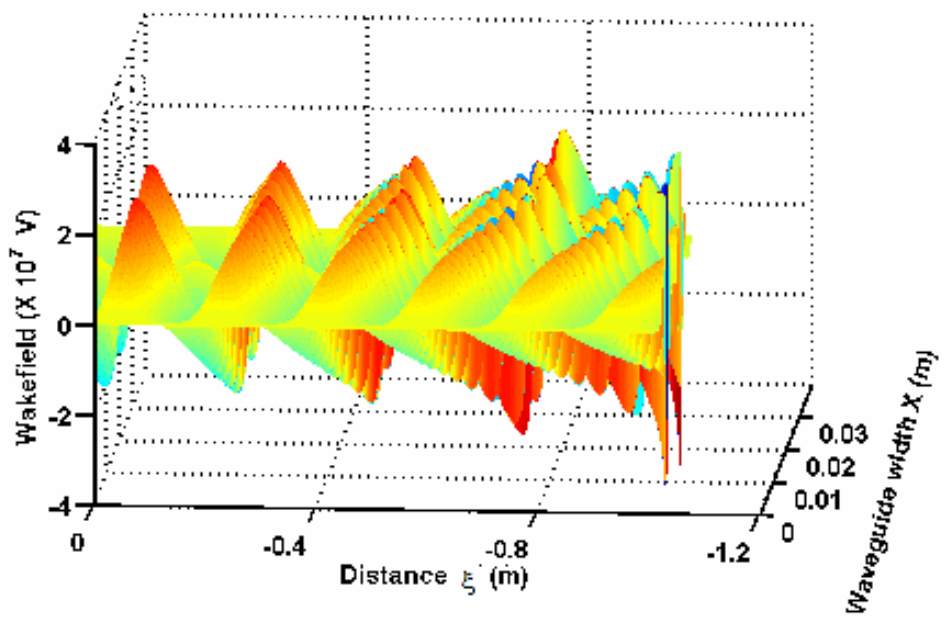

Figure 21. Profile of wakefield corresponding to Fig.20, showing its cutoff around maximum distance of $\xi=3.7 \mathrm{~L}$ from the microwave pulse.

These results suggest that the microwave of higher frequency should be employed for avoiding any instability in the system and the wakefield can be effectively used for the purpose of particle acceleration. The present mechanism of wakefield generation can be realized experimentally if we use wider waveguide filled with higher plasma density. The high density plasma can be produced in the waveguide under the action of Electron Cyclotron Resonance (ECR). However, under such situation short microwave pulses would be more effective in order to resonantly excite the plasma wake wave.

\section{Concluding remarks}

The electromagnetic waves were classified based on their frequency and a small region $\left(10^{11} \mathrm{~Hz}\right.$ to $\left.\sim 10^{13} \mathrm{~Hz}\right)$ that remained the last unexplored region was introduced as the $\mathrm{THz}$ rays. While explaining the propagation of EM waves, it was shown that their propagation velocity depends on the properties of the medium, and unlike the cases of vacuum and dielectrics, their electric field and magnetic field vectors do not remain in phase in the case of conductors. Very fascinating phenomenon of skin depth was discussed in the conductors where the wave vector was found to become a complex quantity and its imaginary part led to the attenuation of the wave. It was mentioned that the EM waves can propagate through the plasma medium if their frequency is larger than the plasma frequency. In the case of plasmas, another interesting phenomenon of wakefield excitation by the laser or microwave pulses was talked about in detail. Two case studies were conducted using the laser pulses and microwave pulses with different envelopes. It was shown that moderate intensity microwave pulses can also generate the wakefield effectively and accelerate the particles to sufficiently large energies. Moreover, the high cost laser systems can be replaced with microwave systems if the microwave pulses can be tailored properly. 


\section{Applications and future prospects}

The electromagnetic wave and plasma interaction has diverse applications in different fields such as nuclear fusion, particle acceleration, heating of ionospheric and laboratory plasmas by radio waves etc. along with controlled fusion applications to ITER (International Thermonuclear Experimental Reactor), frequency upshifting, resonance absorption, laser focusing and defocusing, material processing, generation of $\mathrm{X}$-ray, $\mathrm{THz}$ and microwave radiations, higher order harmonic generation, laser filamentation etc. With the inclusion of plasma, the performance of some devices such as backward wave oscillator (BWO), travelling wave tube (TWT) amplifiers, gyrotrons and other microwave tubes have been found to increase.

The use of laser plasma accelerators has been made in radioisotope production through $(\gamma, n)$ reactions with laser accelerated electron bunches in the range tens of $\mathrm{MeV}$ [92, 93]. The short pulse nature and high charge of the accelerated bunches also has applications in the production of coherent $\mathrm{THz}$ radiation, which is achieved when femtosecond electron bunches cross the plasma vacuum boundary and emit transition radiation [94 - 96]. The generation of $\mathrm{THz}$ radiation has interesting applications in nonlinear $\mathrm{THz}$ spectroscopy, material characterization, imaging, topography, remote sensing, chemical and security identification $[97,98]$ etc. Another application of accelecerated electron beams / bunches is in the generation of femtosecond $\mathrm{X}$-ray pulses produced by the betatron radiation emitted when the electron beam propagates through the plasma. By making an array of nanoholes on an alumina target, $\mathrm{X}$-ray emission from laser produced plasma can be greatly enhanced even in soft $\mathrm{X}$-ray energy regions $(<0.25 \mathrm{keV})$. The enhancement increases as the ionization level of $\mathrm{Al}$ becomes higher and the X-ray wavelength becomes shorter. Over 50 fold enhancement was obtained at a soft X-ray wavelength around $6 \mathrm{~nm}$, which corresponds to the emission from $\mathrm{Al}^{8+, 9+}$ ions. X-ray pulse duration was $17 \mathrm{ps}$, which is much shorter than that obtained by using the prepulse technique [99]. Towards the generation of other types of electromagnetic radiation, Tripathi and Liu [82] have proposed a dielectric-lined waveguide for the free-electron laser emission in millimeter wavelength band. Farokhi et al. [83] have presented a linear theory for a free electron laser with a three-dimensional helical wiggler and axial magnetic field in the collective regime in a configuration consisting of an annular electron beam propagating inside a cylindrical waveguide. For the generation of high power (140 MW) subnanosecond (75 ps) microwave pulses in the range of $38-150 \mathrm{GHz}$, Yalandin et al. [84] have done experiments on coherent stimulated radiation from intense, subnanosecond electron bunches moving through a periodic waveguide and interacting with a backward propagating TM01 wave. Hayashi et al. [85] have also designed a two-stage ferroelectric electron gun and a peak power of 5.9 MW microwave radiation was observed when a $100 \mathrm{~A} 450 \mathrm{kV}$ electron beam was used.

Research directed towards the development of high power electromagnetic radiation sources accounts for much of the current interest in the plasma filled waveguides. Plasma filled waveguides may also be used for the transportation of electromagnetic energy and charged particles, and in the basic study of plasma phenomena. In spite of such extensive 
work over the years, the understanding of the physics of wave plasma interaction is still an active area of research, which also finds additional applications in plasma based focused ion beams (FIB), plasma sources for negative ion beams for neutral beam injection, rf-based plasma thrusters, etc. With regard to the particle acceleration, we have carried out analytical and numerical studies on the wakefield excitation by different types of the pulses in a rectangular waveguide filled with homogeneous plasma. Our analyses reveal that moderate intensity $\left(\sim 10^{9} \mathrm{~W} / \mathrm{m}^{2}\right)$ microwave pulses can produce up to $100 \mathrm{MV} / \mathrm{m}$ wakefield in the waveguide if the nanosecond pulses are used. Since the amplitude of resonantly excited wakefield changes at a faster rate with the waveguide width, pulse duration and microwave intensity and it is larger for the smaller waveguide width, longer pulse duration and the higher microwave intensity in the case of rectangular triangular pulse, the significant wakefield can be excited in the waveguide and effective particle acceleration can be achieved with the use of RT pulses for which the parameters can be optimized using the present studies. This theoretical work on the contribution of different microwave and laser pulses for the purpose of particle acceleration and the THz generation [100, 101] shall induce experimentalists to develop rectangular, rectangular-Gaussian, rectangular-triangular, sawtooth and triagular pulses of appropriate lengths for accomplishing various experiments on wakefield generation, particle acceleration, and the $\mathrm{THz}$ generation. Through such efforts our researchers would be able to benfit the society more via the medical, scientific, and technological applications of the subject electromagnetic wave and plasma interaction.

\section{Author details}

Hitendra K. Malik

Department of Physics, Indian Institute of Technology Delhi, New Delhi, India

\section{References}

[1] V. L. Ginzburg, The propagation of electromagnetic waves in plasmas, Pengamon, New York (1970).

[2] N. G. Denisov, Zh. Eksp. Teor. Fiz. 31, 609 (1956); Engi. Transi. Sov. Phys. JETP 4, 544 (1957).

[3] P. Koch and J. Albritton, Phys. Rev. Left. 32, 1420 (1974); J. Aibritton and P. Koch, Phys. Fluids 18, 1136 (1975).

[4] V. B. Gildenburg, Sov. Phys. JETP 18, 1359 (1964).

[5] T. H. Stix, Phys. Fluids 1, 308 (1958).

[6] B. N. Breizman and A. V. Arefiev, Phys. Plasmas 8, 907 (2001);

[7] A. F. Kuckes, Plasma Phys. 10, 367 (1968)

[8] M. N. Rosenbluth and C. S. Liu, Phys. Rev. Lett. 29, 701 (1972).

[9] C. E. Clayton, C. Joshi, C. Darrow and D. Umstadter, Phys. Rev. Lett. 54, 2343 (1985).

[10] W. Yu and Z. -Z. Xu, Phys. Rev. A. 36, 285 (1987).

[11] R. P. H. Chang, M. Porkolab and B. Grek, Phys. Rev. Lett. 28, 206 (1972)

[12] A. B. Kitsenkov, I. Panchenko and K. N. Stepanov, Plasma Phys. 161109 (1974). 
[13] R. Fedosejevs, X. F. Wang and G. D. Tsakiris, Phys. Rev. E 56, 4615 (1997)

[14] C. E. Max and J. Arons Phys. Rev. Lett. 33, 209 (1974)

[15] P. K. Kaw, G. Schmidt and T. Wilcox, Phys. Fluids 16, 1522 (1973)

[16] D. J. Nicholas and S. G. Sajjadi, J. Phys. D: Appl. Phys. 19, 737 (1986)

[17] D. A. Jones, E. L. Kane, P. Lalousis, P. R. Wiles and H. Hora, Appl. Phys. B 27, 157 (1982)

[18] T. Tajima and J. M. Dawson, Phys. Rev. Lett. 43, 267 (1979).

[19] P. Chen, J. M. Dawson, R. W. Huff and T. Katsouleas, Phys. Rev. Lett. 54, 693 (1985).

[20] J. B. Rosenzweig, D. B. Cline, B. Cole, H. Figueroa, W. Gai, R. Konecny, J. Norem, P. Schoessow and J. Simpson, Phys. Rev. Lett. 61, 98 (1988).

[21] J. B. Rosenzweig, P. Schoessow, B. Cole, W. Gai, R. Konecny, J. Norem and J. Simpson, Phys. Rev. A 39, 1586 (1989).

[22] K. Nakajima, D. Fisher, T. Kawakubo, H. Nakanishi, A. Ogata, Y. Kato, Y. Kitagawa, R. Kodama, K. Mirna, H. Shiraga, K. Suzuki, K. Yamakawa, T. Zhang, Y. Sakawa T. Shoji, Y. Nishida, N. Yugarni, M. Downer and T. Tajima, Phys. Rev. Lett. 74, 4428 (1995).

[23] H. K.Malik, S. Kumar and Y. Nishida Electron. Opt. Comm. 280, 417 (2007).

[24] K. V. Lotov, Laser Part. Beams 19, 219 (2001).

[25] A. J. W. Reitsma and D. A. Jaroszynski, Laser Part. Beams 22, 407 (2004).

[26] A. F. Lifschitz, J. Faure, Y. Glinec, V. Malka and P. Mora, Laser Part. Beams 24, 255 (2006).

[27] K. Flippo, B. M. Hegelich, B. J. Albright, I. Yin, D. C. Gautier and S. Letzring, M. Schollmeier, J. Schreiber, R. Schulze, J. C. Fernandez. Laser Part. Beams 25, 3 (2007).

[28] P. V. Nickles, S. Ter-avetisyan, M. Schnuerer, T. Sokollik, W. Sandner, J. Schreiber, D. Hilscher, U. Jahnke, A. Andreev and V. Tikhonchuk, Laser Part. Beams 25, 347 (2007).

[29] C. T. Zhou, M. Y. Yu and X. T. He, Laser Part. Beams 25, 313 (2007).

[30] Y. Nishida, T. Okazaki, N. Yugami and T. Nagasawa, Phys. Rev. Lett. 66, 2328 (1991).

[31] D. W. Aossey, J. E. Williams, H.-S. Kim, J. Cooney and Y.-C. Hsu and K. E. Lonngren, Phys. Rev. E 47, 2759 (1993).

[32] B. B. Balakirev, V. I. Karas, I. V. Karas and V. D. Levchenko, Laser Part. Beams 19, 597 (2001).

[33] T. B. Zhang, J. L. Hirshfield, T. C. Marshall and B. Hafizi, Phys. Rev. E 56, 4647 (1997) .

[34] S. Y. Parka and J. L. Hirshfield, Phys. Rev. E 62, 1266 (2000).

[35] C. Jing, W. Liu, I. Xiao, W. Gai and P. Schoessow, Phys. Rev. E 68, 016502 (2003).

[36] Y. Nishida, S. Kusaka and N. Yugami, Physica Scripta T52, 65 (1994).

[37] Z. M. Sheng, K. Mima, Y. Sentoku, K. Nishihara and J. Zhang, Phys. Plasmas 9, 3147 (2002).

[38] H.K. Malik, J. Appl. Phys. 104, 053308 (2008).

[39] R. J. Kingham and A. R. Bell, Phys. Rev. Lett. 79, 4810 (1997).

[40] N. E. Andreev, M. V. Chegotov and M. E. Veisman, IEEE Trans. Plasma Sci. 28, 1098 (2000).

[41] B. Cros, C. Courtois, G. Malka, G. Matthieussent, J. R. Marques, F. Dorchies, F. Amiranoff, S. Rebibo, G. Hamoniaux, N. Blanchot and J. L. Miquel, IEEE Trans. Plasma Sci. 28, 1071 (2000). 
[42] P. Sprangle, B. Hafizi, J. R. Peñano, R. F. Hubbard, A. Ting, C. I. Moore, D. F. Gordon, A. Zigler, D. Kaganovich and T. M. Antonsen Jr., Phys. Rev. E 63, 056405 (2001).

[43] P. Manz, M. Ramisch and U. Stroth, Phys. Rev. Lett. 103, 165004 (2009).

[44] T. Happel, F. Greiner, N. Mahdizadeh, B. Nold, M. Ramisch and U. Stroth, Phys. Rev. Lett. 102, 255001 (2009).

[45] U. Stroth, Plasma Phys. Control. Fusion 40, 9 (1998).

[46] U. Stroth, F. Greiner, C. Lechte, N. Mahdizadeh, K. Rahbarnia and M. Ramisch, Phys. Plasmas 11, 2558 (2004).

[47] P. Manz, M. Ramisch, U. Stroth, V. Naulina and B. D. Scott, Plasma Phys. Control. Fusion 50, 035008 (2008).

[48] M. Ramisch, E. Häberle, N. Mahdizadeh and U. Stroth, Plasma Sources Sci Technol. 17, 024007 (2008).

[49] F. Aziz and U. Stroth, Phys. Plasmas 16, 032108 (2009).

[50] H. D. Hochheimer, K. Weishaupt and M. Takesada, J. Chem. Phys. 105, 374 (1996).

[51] F. Widulle, J. T. Held, M. Huber, H. D. Hochheimer, R. T. Kotitschke and A. R. Adams, Rev. Sci. Instrum. 68, 3992 (1997).

[52] A. Asenbaum, O. Blaschko and H. D. Hochheimer, Phys. Rev. B 34, 1968 (1986).

[53] A. B. Garg, V. Vijayakumar, B. K. Godwal, A. Choudhury and H. D. Hochheimer, Solid State Comm. 142, 369 (2007).

[54] R. Singh and M. P. Bora, Phys. Plasmas 7, 2335 (2000).

[55] N. Singh and R. Singh, Phys. Plasmas 11, 5475 (2004).

[56] R. Singh, V. Tangri, P. Kaw and P. N. Guzdar, Phys. Plasmas 12, 092307 (2005).

[57] N. Chakrabarti, R. Singh, P. K. Kaw and P. N. Guzdar, Phys. Plasmas 14, 052308 (2007).

[58] M. Starodubtsev and C. Krafft, Phys. Rev. Lett. 83, 1335 (1999)

[59] M. Starodubtsev and C. Krafft, Phys. Plasmas 6, 2598 (1999)

[60] A. K. Attri, U. Kumar and V. K. Jain, Nature 411, 1015 (2001).

[61] S. H. Kim, E. Agrimson, M. J. Miller, N. D'Angelo, R. L. Merlino and G. I. Ganguli, Phys. Plasmas 11, 4501 (2004).

[62] S. C. Sharma and M. P. Srivastava, Phys. Plasmas 8, 679 (2001).

[63] Y. Sakawa, C. Joshi, P. K. Kaw, F. F. Chen and V. K. Jain, Phys. Fluids B 5, 1681 (1993).

[64] A. Neogi and R. K. Thareja, Phys. Plasmas 6, 365 (1999).

[65] T. A. Peyser, C. K. Manka, B. H. Ripin and G. Ganguli, Phys. Fluids B 4, 2448 (1992).

[66] C. J. McKinstrie and E. A. Startsev, Phys. Rev. E 54, R1070 (1996).

[67] A. Pukhov, Phys.Rev. Lett. 86, 3562 (2001).

[68] M. Y. Yu, W. Yu, Z. Y. Chen, J. Zhang, Y. Yin, L. H. Cao, P. X. Lu and Z. Z. Xu, Phys. Plasmas, 10, 2468 (2003).

[69] H. Suk, J. Appl. Phys. 91, 487 (2002).

[70] Y. Sentoku, T. E. Cowan, A. Kemp and H. Ruhl, Phys. Plasmas 10, 2009 (2003).

[71] K. P. Singh and V. K. Tripathi, Phys. Plasmas 11, 743 (2004).

[72] M. Kado, H. Daido, A. Fukumi, Z. Li, S. Orimo, Y. Hayashi, M. Nishiuchi, A. Sagisaka, K. Ogura, M. Mori, S. Nakamura, A. Noda, Y. Iwashita, T. Shirai, H. Tongu, T. Takeuchi, A. Yamazaki, H. Itoh, H. Souda, K. Nemoto, Y. Oishi, T. Nayuki, H. Kiriyama, S. Kanazawa, M. Aoyama, Y. Akahane, N. Inoue, K. Tsuji, Y. Nakai, Y. 
Yamamoto, H. Kotaki, S. Kondo, S. Bulanov, T. Esirkepov, T. Utsumi, A. Nagashima, T. Kimura and K. Yamakawa, Laser Part. Beams 24, 117 (2006).

[73] A. Karmakar and A. Pukhov, Laser Part. Beams 25, 371 (2007).

[74] J. J. Xu, Q. Kong, Z. Chen, P. X. Wang, W. Wang, D. Lin and Y. K. Ho, Laser Part. Beams 25,253 (2007).

[75] Y. Nishida and T. Shinozaki, Phys. Rev. Lett. 65, 2386 (1990).

[76] Y. Nishida and N. Sato, Phys. Rev. Lett. 59, 653 (1987).

[77] Y. Nishida, M. Yoshizumi and R. Sugihara, Phys. Fluids 28, 1574 (1985).

[78] C.G. Durfee III, A. R. Rundquist, S. Backus, C. Herne, M. M. Murnane and H. C. Kapteyn, Phys. Rev. Lett. 83, 2187 (1999).

[79] X. Letartre, C. Seassal, C. Grillet, P. Rojo-Romeo, P. Viktorovitch, M. Le V. d'Yerville, D. Cassagne and C. Jouanin, Appl. Phys. Lett. 79, 2312 (2001).

[80] J. L. Hirshfield, M. A. LaPointe, A. K. Ganguly, R. B. Yoder and C. Wang Phys. Plasmas 3, 2163 (1996).

[81] E. Carlsten, Phys. Plasmas 9, 5088 (2002).

[82] V. K. Tripathi and C. S. Liu, IEEE Trans. Plasma Sci. 17, 583 (1989).

[83] B. Farokhi, Z. Family and B. Maraghechi, Phys. Plasmas 10, 2566 (2003).

[84] M. I. Yalandin, V. G. Shpak, S. A. Shunailov, M. R. Oulmaskoulov, N. S. Ginzburg, I. V. Zotova, Y. V. Novozhilova, A. S. Sergeev, A. D. R. Phelps, A. W. Cross, S. M. Wiggins and K. Ronald, IEEE Trans. Plasma Sci. 28, 1615 (2000).

[85] Y. Hayashi, X. Song, J. D. Ivers, D. D. Flechtner, J. A. Nation and L. Schächter, IEEE Trans. Plasma Sci. 29, 599 (2001).

[86] R. B. Yoder, T. C. Marshall and J. L. Hirshfield, Phys. Rev. Lett. 86, 1765 (2001).

[87] S. Kumar and H. K. Malik, J. Plasma Phys. 72, 983 (2006).

[88] H. K. Malik, Opt. Comm. 278, 387 (2007).

[89] A.K. Aria and H. K. Malik, The Open Plasma Phys. J. 1, 1 (2008).

[90] A.K. Aria, H. K. Malik and K.P. Singh, Laser Part. Beams 27, 41 (2009).

[91] S.V. Bulanov, T.J. Esirkepov, N.M. Naumova, F. Pegoraro, I.V. Pogorelsky, A.M. Pukhov, IEEE Trans. Plasma Sci. 24, 393 (1996).

[92] W. P. Leemans, D. Rodgers, P. E. Catravas, C. G. R. Geddes, G. Fubiani, E. Esarey, B. A. Shadwick, R. Donahue and A. Smith, Phys. Plasmas 8, 2510 (2001).

[93] M. I. K. Santala, M. Zepf, F. N. Beg, E. L. Clark, A. E. Dangor, K. Krushelnick, M. Tatarakis, I. Watts, K. W. D. Ledingham, T. McCanny, I. Spencer, A. C. Machacek, R. Allott, R. J. Clarke and P. A. Norreys, Appl. Phys. Lett. 78, 19 (2001).

[94] W. P. Leemans, C. G. R. Geddes, J. Faure, C. Tóth, J. V. Tilborg, C. B. Schroeder, E. Esarey, G. Fubiani, D. Auerbach, B. Marcelis, M. A. Carnahan, R. A. Kaindl, J. Byrd and M. Martin, Phys. Rev. Lett. 91, 074802 (2003).

[95] C. B. Schroeder, E. Esarey, J. V. Tilborg and W. P. Leemans, Phys. Rev. E 69, 016501 (2004).

[96] W. P. Leemans, J. V. Tilborg, J. Faure, C. G. R. Geddes, C. To'th, C. B. Schroeder, E. Esarey, G. Fubiani and G. Dugan, Phys. Plasmas 11, 2899 (2004).

[97] Y. C. Shen, T. W. P. F. Today, B. E. Cole, W. R. Tribe, and M. C. Kemp, Appl. Phys. Lett. 86, 241116 (2005). 
[98] H. Zhong, A. Redo-Sanchez, and X.-C. Zhang, Opt. Express 14, 9130 (2006).

[99] T. Nishikawa, H. Nakano, N. Uesugi, M. Nakao and H. Masuda, Appl. Phys. Lett. 75, 4079 (1999).

[100] A.K. Malik, H.K. Malik and S. Kawata, J. Appl. Phys. 107, 113105 (2010).

[101] A.K. Malik, H.K. Malik and Y. Nishida, Phys. Lett. A 375, 1191 (2011). 